(C) 2017, American Psychological Association. This paper is not the copy of record and may not exactly replicate the final, authoritative version of the article. Please do not copy or cite without authors permission. The final article will be available, upon publication, via its DOI: $10.1037 / p s p p 0000186$

Paper accepted at Journal of Personality and Social Psychology: Personality Processes and Individual Differences. Cite As:

Dejonckheere, E., Mestdagh, M., Houben, M., Erbas, Y., Pe, M., Bastian, B., Koval, P., Brose, A., \& Kuppens, P. (2018). The bipolarity of affect and depressive symptoms. Journal of Personality and Social Psychology, DOI: 10.1037/pspp0000186 


\title{
The Bipolarity of Affect and Depressive Symptoms
}

Egon Dejonckheere ${ }^{1}$, Merijn Mestdagh ${ }^{1}$, Marlies Houben ${ }^{1}$, Yasmine Erbas ${ }^{1}$, Madeline Pe ${ }^{1}$, Brock Bastian ${ }^{2}$, Peter Koval ${ }^{2}$, Annette Brose $^{3}$, \& Peter Kuppens ${ }^{1}$

\author{
${ }^{1}$ KU Leuven - Faculty of Psychology and Educational Sciences \\ ${ }^{2}$ University of Melbourne - School of Psychology \\ ${ }^{3}$ Humboldt-Universität zu Berlin - Institut für Psychologie
}

\begin{abstract}
Author note
The research leading to the results reported in this manuscript was supported by the Research Fund of KU Leuven (GOA/15/003; OT/11/031), by the Interuniversity Attraction Poles program financed by the Belgian government (IAP/P7/06) and by the Australian Research Council Discovery grant awarded to Brock Bastian and Peter Kuppens (DP140103757).

Correspondence concerning this article should be addressed to Egon Dejonckheere, Faculty of Psychology and Educational Sciences, KU Leuven, Tiensestraat 102, Leuven, 3000, Belgium. E-mail: egon.dejonckheere@kuleuven.be
\end{abstract}




\begin{abstract}
People differ in the extent to which they experience positive (PA) and negative affect (NA) rather independently or as bipolar opposites. Here, we examine the proposition that the nature of the relation between positive and negative affect in a person's emotional experience is indicative of psychological well-being, in particular the experience of depressive symptoms, typically characterized by diminished positive affect (anhedonia) and increased negative affect (depressed mood). In three experience sampling studies, we examine how positive and negative affective states are related within people's emotional experience in daily life and how the degree of bipolarity of this relation is associated with depressive symptom severity. In Study 1 and 2, we show both concurrently and longitudinally that a stronger bipolar PA-NA relationship is associated with, and in fact is predicted by, higher depressive symptom severity, even after controlling for mean levels of positive and negative affect. In Study 3, we replicate these findings in a daily diary design, with the two conceptually related main symptoms of depression, sadness and anhedonia, as specific manifestations of high NA and low PA, respectively. Across studies, additional analyses indicate these results are robust across different timescales and various PA and NA operationalizations and that affective bipolarity shows particular specificity towards depressive symptomatology, in comparison with anxiety symptoms. Together, these findings demonstrate that depressive symptoms involve stronger bipolarity between positive and negative affect, reflecting reduced emotional complexity and flexibility.
\end{abstract}

Keywords: positive affect, negative affect, affective bipolarity, affective independence, depressive symptoms 


\section{The Bipolarity of Affect and Depressive Symptoms}

Emotional disturbances and abnormal patterns in affect are prominently apparent in the experience of depressive symptoms. These symptoms are highly prevalent in the general population and are known to significantly impact people's psychological well-being (Ferrari et al., 2013; Shim, Baltrus, Ye, \& Rust, 2011). Depressive symptomatology is centrally characterized by increased levels of negative affect (NA) combined with decreased levels of positive affect (PA; Clark \& Watson, 1991; Watson, Clark, \& Carey, 1988). Traditionally, PA and NA have been studied in isolation in relation to depression (e.g. Klein, 1984; Watson \& Clark, 1984). In the present paper, we argue that beyond altered mean levels of positive and negative affect, depressive symptomatology also involves the experience of PA and NA becoming more mutually exclusive, with negative feelings implying a lack of positive feelings and vice versa, reflected in a more negative relation between the two.

\section{The Relationship between Positive and Negative Affect}

How feeling good and feeling bad are related in (normal) human experience is a topic of long and intense debate, central to the psychology of affect (Nowlis \& Nowlis, 1956; Schmukle, Egloff, \& Burns, 2002; Watson \& Clark, 1997). Are positive and negative affect bipolar opposites of one and the same dimension in human experience? Or do these affective states rather make up two independent dimensions? This question is anything but trivial, as its answer guides our fundamental understanding of the nature of emotions, their causes and consequences (Diener, 1999; Russell \& Carroll, 1999).

In their Circumplex Model of Affect, Russell and colleagues (Barrett \& Russell, 1998; Russell, 1979, 1980) propose that all emotional states can fundamentally be characterized in terms of two psychologically irreducible qualities, valence and arousal. In this model, the valence of emotion is conceptualized as a bipolar continuum stretching between pleasant and unpleasant states, with PA (e.g. happiness) and NA (e.g. sadness) being the mutually 
exclusive poles of this dimension (Russell \& Carroll, 1999). Following these assumptions, positive and negative feelings operate reciprocally, such that increases in PA are considered functionally equivalent to decreases in NA, and vice versa (Russell, 1979). Arousal, on the other hand, lies orthogonal to the valence dimension and describes the degree of activation of an emotional state, ranging from sleepiness to being highly aroused.

In turn, other scholars have argued that PA and NA are fundamentally orthogonal (Diener \& Emmons, 1985; Thayer, 1989; Warr, Barter, \& Brownbridge, 1983; Watson, Wiese, Vaidya, \& Tellegen, 1999). In their two-factor structure of the affective space, Watson and Tellegen (1985) proposed PA (e.g. excitement) and NA (e.g. distress) as activated states, operating largely independently from each other ${ }^{1}$. Both PA and NA are understood to have their own bio-behavioral basis, with positive affect relating to approach motivation and behavior and negative affect relating to withdrawal or avoidance (Watson et al., 1999) ${ }^{2}$. Although evidence for both affective bipolarity and independence exists (e.g. Larsen, McGraw, \& Cacioppo, 2001; Reich, Zautra, \& Davis, 2003; Russell \& Carroll, 1999; Watson et al., 1988), decades of research suggest these different viewpoints may not be necessarily mutually exclusive. In fact, studies show that the degree to which affect is bipolar versus independent may vary as function of a number of factors, effectively reconciling both seemingly conflicting perspectives (Watson, 1988).

First, the way positive and negative affect are measured not unexpectedly affects conclusions of bipolarity versus independence (Russell \& Carroll, 1999). Since both affective

\footnotetext{
${ }^{1}$ The contradiction between these two viewpoints is less sharp than appears on first sight, however. As PA and NA in the latter model are considered to be high in arousal (i.e. activated states), it was concluded that this perspective is largely equivalent to the former, reflecting a $-45^{\circ}$ rotation of the model proposed by Russell and colleagues (Barrett \& Russell, 1998; Yik, Russell, \& Barrett, 1999). Watson et al. (1999) later renamed their PA and NA dimensions to positive and negative activation to distinguish their constructs from Russell's (1980) original positive and negative affect.

${ }^{2}$ Note that other affective models have been proposed to explain the relation between positive and negative emotionality (e.g. Cacioppo, Gardner, \& Berntson, 1997; Diener \& Iran-Nejad, 1986; Schimmack, 2001). However, as these theories do not rely on the affective space in their predictions, a comprehensive discussion is beyond the scope of this paper (see Larsen et al. [2001] for an extensive review).
} 
states are not consensually defined, and thus no agreement exists upon which emotion terms should make up the scales, a broad variety of affective instruments is available (e.g. Bradburn, 1969; Nowlis \& Nowlis, 1956; Watson, Clark, \& Tellegen, 1988), with each scale yielding different results on the strength of the PA-NA relationship. In particular, the degree to which PA and NA items (a) are really semantic antonyms and (b) cover different levels of arousal is known to be of importance in shaping their relation (Barrett \& Russell, 1998).

Second, the assessed time frame also alters the observed association (Diener \& Emmons, 1985). PA and NA become more negatively correlated as the examined time period is compressed, with momentary assessments generally showing a substantial bipolar relation. In the same vein, positive and negative affect are known to show a considerable negative relation on a state-level, while trait PA and NA tend to be unrelated (Schmukle et al., 2002; Tellegen, 1985).

But perhaps most importantly, the relationship between positive and negative affect has been found to vary substantially across individuals (e.g. Brose, Voelkle, Lövdén, Lindenberger, \& Schmiedek, 2015; Feldman, 1995; Pitzer \& Bergeman, 2014; Potter, Zautra, \& Reich, 2000; Rafaeli, Rogers, \& Revelle, 2007): while some people experience PA and NA rather independently, for others these affective states are experienced as mutually exclusive extremes of a single dimension ${ }^{3}$.

Individual Differences in the Within-person Relationship between Positive and Negative Affect.

Individual differences in the relationship between PA and NA have been studied previously under different names. Rafaeli and collegues (2007) for example, introduced the term affective synchrony to explain between-person variability in the within-person

\footnotetext{
${ }^{3}$ Still other factors that are known to influence this affective relationship are the response type format (Russell \& Carroll, 1999; Warr et al., 1983; Watson, 1988), measurement error (Green et al., 1993), and the level of analysis (within- versus between-person level; Brose, Voelkle, et al., 2015).
} 
association between positive and negative affect. They assessed the temporal stability of this relation and examined how the tendency towards affective independence is associated with individual differences in the cognitive representation of the self and of emotions. Feldman (1995) used the term valence focus to refer to how people differ in the degree to which they attend to the hedonic component of an emotional experience. She illustrated how individuals high in valence focus do not only exhibit a more bipolar experience of affect at the withinperson level, but are also less able to discriminate between anxious and depressed moods (see also Erbas, Ceulemans, Koval, \& Kuppens, 2015). Research on emotional dialecticism (Grossmann, Huynh, \& Ellsworth, 2015; Lindquist \& Barrett, 2008) investigates how affect is structured in relation to factors such as age (e.g. Carstensen et al., 2011; Ready, Åkerstedt, \& Mroczek, 2011), gender (e.g. Bagozzi, Wong, \& Yi, 1999) and cultural differences (e.g. Grossmann et al., 2015). For extensive reviews, see Grühn, Lumley, Diehl, \& Labouvie-vief (2013) and Brose, de Roover, Ceulemans, \& Kuppens (2015) .

Whether and how individual differences in the relation between positive and negative affect relate to psychological well-being remains nevertheless underexplored. With average levels of PA and NA being so centrally involved in well-being and mood disorders, it is not unlikely that how they are mutually structured may also play a central role in people's mental health. While there is some indirect evidence for this claim, few studies, however, directly investigated the meaningfulness of these individual differences in relation to psychological well-being. Here, we propose that the within-person relation between positive and negative affect may be indicative of emotion dysregulation, in particular as it can be observed in the experience of depressive symptoms.

\footnotetext{
${ }^{4}$ Note that researchers previously also used the term co-occurrence to refer to the correlation between PA and NA (e.g. in the light of mixed emotional experiences). A correlational approach, however, tells us little about what extent oppositely valenced states are simultaneously present in the moment (Larsen, Hershfield, Stastny, \& Hester, 2017). More appropriate measures have been proposed to make claims about actual co-occurrence of PA and NA (e.g. Schimmack, 2001).
} 


\section{Individual Differences in the Within-person Relationship between Positive and Negative}

\section{Affect and Depressive Symptoms.}

Depressive complaints are heterogeneous in nature, but they at least involve the experience of one of the following mood-related symptoms: depressed mood and/or anhedonia (DSM-5; American Psychiatric Association, 2013). Depression’s first core symptom, depressed mood, encompasses feelings of prolonged sadness and is considered a particular manifestation of the general construct of negative affect (Watson \& Clark, 1984). Experiencing NA is not specific to depressive symptomatology, but rather encompasses general psychological distress, shared with other types of (vulnerability to) psychopathology, such as anxiety disorders (Clark \& Watson, 1991). Depression's second key symptom, anhedonia, reflects a significant diminishment in pleasure or interest and as such refers to an individual's lack of positive affect (Watson, Clark, \& Carey, 1988). It is associated with a deficit in motivation to engage in typically rewarding behavior (Klein, 1984) and impaired reward responsiveness (Treadway \& Zald, 2013). Low levels of positive affect distinguish depressive symptomatology from other complaints (e.g. anxiety-related symptoms), where the motivation to pursue and the ability to enjoy positive stimuli generally remains unaltered (Clark \& Watson, 1991).

The fact that depression's key symptoms, depressed mood and anhedonia, show conceptual relations with elevated negative and diminished positive affect, respectively, raises questions about the nature of the affective relationship in the experience of depressive symptoms: is depressive symptomatology merely characterized by altered mean levels of PA and NA, or does it additionally involve changes in the structure of the affective space (i.e. how PA and NA mutually relate)? Here, we argue that positive and negative affect may additionally become more polarized, characterized by a stronger inverse relationship between the two. 
An integration of various research lines leads to the idea that depressive symptoms could be associated with a more bipolar experience of positive and negative affect. First, in the context of their Dynamic Model of Affect (DMA; Davis, Zautra, \& Smith, 2004; Zautra, Affleck, Tennen, Reich, \& Davis, 2005; Zautra, Berkhof, \& Nicolson, 2002; Zautra, Potter, \& Reich, 1997), Zautra and colleagues argue, and demonstrate empirically, that the contextual factor of stress ${ }^{5}$ may impact the relationship between experienced positive and negative affect. They explain that distressing conditions increase the need for heuristic, undifferentiated emotional processing, favoring ad-hoc decision making over rigorous deliberation (Reich et al., 2003). Thus, as cognitive resources become limited as a consequence of stress, emotional simplification is desired, fusing PA and NA to become a single bipolar dimension. Although the DMA does not explicitly address individual differences in the PA-NA relationship, this model does suggest that individuals who are chronically distressed would exhibit stronger bipolarity in their affective experiences (Davis et al., 2004). If anything, depressive symptoms involve distress, are burdensome (Fossati, Ergis, \& Allilaire, 2002; Gotlib \& Joormann, 2010; Kircanski, Joormann, \& Gotlib, 2012), and are likely to constrain people's cognitive resources (e.g. rumination; Christopher \& Macdonald, 2005; DeBattista, 2005; Rydmark et al., 2006), potentially introducing a bipolar affective experience in light of these symptoms.

Second, although the DMA mostly links general distress to affective bipolarity, and thus does not specifically focus on depressive symptomatology, additional theoretical arguments exist why depressive symptoms in particular may constrain the affective space. Based on Clark and Watson's Tripartite Model of Anxiety and Depression (1991), for instance, one may expect that affective bipolarity is particularly prominent in (the distress

\footnotetext{
${ }^{5}$ In the DMA, the concept of stress is defined rather broadly, with stressors ranging from artificial lab stimuli (e.g. cold suppressor tasks or speech tests [Zautra, Reich, Davis, Potter, \& Nicolson, 2000]), to frequent experiences of minor stressors or daily hassles (e.g. Zautra et al., 2002), to major life events (e.g. bereavement of a beloved one [Coifman, Bonanno, \& Rafaeli, 2007] or dealing with chronic pain [Potter, Zautra, \& Reich, 2000]). Hence, in this context, stress more closely relates to broad levels of distress or high negative affect, rather than the specific physiological reactions in response to aversive external stimuli.
} 
associated with) the experience of depressive, but not anxiety-related symptoms (Watson \& Kendall, 1989). Although both depressive and anxiety symptoms typically involve high negative affect (indicating distress), the tripartite model posits that it is low PA, or anhedonia, in particular that differentiates depression from anxiety. In anxiety, the combination of unaffected PA states with high levels of NA suggests both affective states may still operate independently, as one state can change without changes in the other. In contrast, in depressed individuals these emotional states are typically both altered in an opposite direction (i.e. high NA, low PA), suggesting these states operate reciprocally (Barlow, Chorpita, \& Turosvsky, 1996), with increases in NA being functionally equivalent to decreases in PA, and vice versa. Although the tripartite model originally relied on between-person data, valuable within-person generalizations have been proposed, with several researchers hinting at a modified, more inverse, affective within-person relation in depression, but not anxiety (Barlow et al., 1996; Watson \& Kendal, 1989) $)^{6}$.

A third rationale why the experience of depressive symptoms may involve a more bipolar emotional life follows from depressed people's impaired goal conceptions. In their Control-Process Model of Affect, Carver and Scheier (1990) argue that the valence of an affective experience is a function of the speed of one's goal attainment. For each goal, people are believed to have an (unconscious) standard about the speed with which they aim to achieve that goal. For a specific goal, affect is understood to be bipolar: quicker progress than what is deemed expected creates positive affect, slower goal attainment is associated with negative affect (Carver, 2004, 2006). People, however, generally pursue multiple goals in life, leading PA and NA to possibly operate relatively independent from each other (Carver \&

\footnotetext{
${ }^{6}$ Both references indirectly speculated about an altered affective relation in depression (in contrast to anxiety), following a similar rationale as outlined above, but had no data to support their claim (Reich et al., 2003). Williams, Peeters, and Zautra (2004) aimed to test this idea and surprisingly found evidence for affective bipolarity in anxiety, but not depression. In their analyses, however, these researchers relied on between-person differences to compute a PA-NA correlation for each group, making generalizations about the structure affect within an individual inappropriate (Brose, Voelkle, et al., 2015).
} 
Scheier, 1990). Individuals high in depression, however, not only have fewer goals, they are also less specific (e.g. I want to be happy; Carver \& Ganellen, 1983; Dickson, Moberly, O’Dea, \& Field, 2016). As a consequence, these very broad, general objectives do not only make it harder for depressed people to achieve their goals (and hence create negative affect), this lack of specificity may also lead their emotional lives to be more bipolar.

Finally, based on the previously cited literature on valence focus and affective synchrony, a strong within-person negative correlation between PA and NA can be regarded as a sign of emotional inflexibility, as it hampers the experience of subtle states in response to ambiguous or mixed events (Feldman, 1995; Rafaeli et al., 2007). If anything, depressive symptomatology is characterized by rigid emotional responding (Houben, Van Den Noortgate, \& Kuppens, 2015; Kuppens et al., 2012; Pe et al., 2015). Combined, these lines of evidence suggest that the emotional maladjustment as expressed in depressive symptoms should involve increased bipolarity of positive and negative affect.

If this is indeed the case, an important next question is whether depressive symptomatology precedes more bipolar affectivity, or vice versa. Based on the DMA, one could argue that the stress endured with depressive symptoms may gradually push people's affective system more towards bipolarity (e.g. Zautra et al., 2000). The opposite may also be true, however, with individuals whose affective experience is more bipolar being at greater risk of developing depression, since they lack a buffer of positive affect when experiencing high levels of negative affect (e.g. van de Leemput et al., 2014), leading to a cycle of prolonged negative mood and anhedonia.

\section{The Current Study}

The goal of the present research is (a) to chart individual differences in the relationship between positive and negative affect and (b) to investigate how such differences are related to, and (c) are predictive for, or are predicted by, people's levels of depressive symptomatology. 
We expect higher depressive symptom severity to be associated with a more inverse (i.e. bipolar) relationship between positive and negative affect.

In Study 1, we examine the association between the momentary PA-NA relation and depressive symptoms concurrently in an undergraduate sample that is stratified on these symptoms. In Study 2, we use a new, similarly constructed sample to investigate the relationship between bipolarity of momentary affect and depressive symptoms both concurrently and longitudinally, allowing us to explore the temporal direction of the relation between the two. Finally, in Study 3, we aim to replicate our concurrent results with data from a daily diary design. In this study, participants' within-person relationship is established between sadness and anhedonia, the two main symptoms of depression, conceptually related to high NA and low PA, respectively. Across studies, we examine the effect of two significant factors known to impact the affective relationship, (a) how PA and NA are operationalized and (b) the time frame in which these affective states are assessed. Given their significance in determining the strength of the affective relationship, it is important to consider how these factors may interact with the association between affective bipolarity and depressive symptoms. Finally, we will examine to what extent affective bipolarity is specific for depressive symptoms in comparison to anxiety-related complaints.

To our knowledge, this is the first study to systematically examine the relation between this crucial aspect of people's emotional experience and depressive symptoms. Going beyond mere mean level of affect, we intend to examine how this structural aspect of people's positive and negative affective experience may be altered when reporting depressive symptomatology. From a theoretical perspective, this study can inform the debate on how PA and NA are related by investigating this relation at a within-person level, stimulating affective scientists to reconsider a fully fixed, invariant perspective on the affect relationship by also taking into account individual differences. From a clinical perspective, looking further than 
average levels of depressed mood and anhedonia will foster our understanding of how these two key components are related in depressive symptomatology, in particular how the experience of negative emotion may go hand in hand with the absence of positive emotion and vice versa.

\section{General Procedure and Statistical Analyses}

Assessing how positive and negative affect are related within an individual's experience implies measuring both constructs at multiple points in time. In the present studies, we will do so by relying on both experience sampling (ESM; Csikszentmihalyi \& Larson, 1987; Study 1 and 2) and daily diary methods (Study 3). These approaches allow us to study dynamic within-person affective processes relatively close to real time (i.e. reducing retrospective biases) throughout participants' daily lives (i.e. highly ecologically valid), providing us with a particularly natural assessment of one's emotional life (Fisher \& To, 2012).

Although the hierarchical data structure created by these methods (multiple measurement occasions per participant) suggests using multilevel regression analyses, we favor a correlational approach to answer the current research questions. Since we aim to determine to what degree PA and NA form a unidimensional construct as a function of depressive symptom severity, we are essentially interested in the reciprocal relationship between these affective states (i.e. their symmetrical dependency). In contrast, in a regression analytic context, we would have to specify one variable (e.g. PA) as a predictor and one variable (e.g. NA) as an outcome variable. Not only is this choice fairly arbitrary (Ong, Zautra, \& Finan, 2017), linear regression in a multilevel context is inherently asymmetrical, meaning that the switch of outcome and predictor may yield different results, which is problematic for the current purpose. This implies that multilevel regression methods are not ideal to test our focal reciprocal relationship. For this reason, we instead calculated a Pearson 
correlation between PA and NA per participant, and examined how this correlation was related to people's level of depressive symptom severity. This correlational approach is in line with the majority of existing literature on the relation between positive and negative affect (e.g. Bagozzi et al., 1999; Carstensen et al., 2011; Davis et al., 2004; Rafaeli et al., 2007)7. Finally, PA-NA correlations partly depend both on participants' mean levels of, and variability in, these affective states. For example, regarding average levels, a participant without depressive symptoms may hardly ever report any negative affect, attenuating that person's observed PA-NA relationship. Similarly, an individual's tendency to formulate extreme responses could impact both that person's mean level of positive and negative affect as well as his reported depressive symptom severity. With respect to individual differences in variability in PA and NA, depressive symptomatology has been repeatedly linked to increased variability in positive and negative emotional responding (Houben et al., 2015; Koval, Pe, Meers, \& Kuppens, 2013) which could also influence the relation between PA and NA (for instance, very low variability could lead to correlations around .0). Thus, to rule out alternative accounts of affective insensitivity, response biases with respect to extreme responding, or the possibility our findings are merely a statistical artefact driven by differences in average levels of, or variability in PA and NA, we will additionally control for these covariates in all reported studies. In this way, we aim to unravel the unique relation between the PA-NA correlation and depressive symptoms, above and beyond these variables.

\section{Ethical Approval}

All studies presented in this article were ethically approved by a research ethics committee. Study 1, entitled Emotion dynamics, executive functions and heart rate variability

\footnotetext{
${ }^{7}$ One way to overcome this asymmetry in a multilevel regression context is to use person-mean standardized variables (Schuurman, Ferrer, de Boer-Sonnenschein, \& Hamaker, 2016). For comparison, we estimated all reported relationships also using multilevel models with within-person standardized outcome and predictor (both PA on NA and NA on PA) and report the results in the Supplementary Materials (Tables 1-3). All models replicate our correlational findings, showing robustness across approaches.
} 
(ML7321 - S53155), and Study 2, entitled The process basis and predictive value of emotion dynamics for psychological well-being (ML8514 - S54567), were approved by the institutional review board of the KU Leuven. Study 3, entitled Mood networks (HREAP 2335) was approved by the UNSW Human Research Ethics Advisory Board (Panel C).

\section{Study 1}

\section{Participants and Prescreening}

Participants were recruited as part of a larger study investigating different aspects of psychological well-being and received $€ 70$ for their participation ${ }^{8}$. In order to detect small to medium effect sizes $(r=.30, \alpha=.05)$, a sample of 100 participants was aimed for.

Using the Center for Epidemiologic Studies Depression Scale (CES-D; Radloff, 1977) an initial pool of 439 Dutch-speaking KU Leuven undergraduates was prescreened in order to recruit a final sample that experienced a wide and balanced range of depressive symptoms (range $=0-52, M=16.39, S D=10.27, \alpha=.92$ ). To achieve this, we adopted a stratified sampling approach (Ingram \& Siegle, 2009) in which the CES-D prescreening range was divided into five approximately equal segments of which we randomly contacted a sample of participants from each segment (range $=0-50, M=19.27 ; S D=12.53$ ).

One student withdrew early and four additional participants were excluded from data analysis due to poor compliance with the ESM protocol (i.e., $<50 \%$ response rate; $n=1$ ) or equipment malfunction ( $n=3$ ), leaving us with a final simple of 95 participants (36 men; $M_{\text {age }}$ $\left.=19.06, S D_{\text {age }}=1.28\right)^{9}$.

\section{Materials and Procedure}

\footnotetext{
${ }^{8}$ Parts of the presented data have been published elsewhere, but no overlap exists with previously published work. In Koval, Ogrinz, et al. (2013) we establish a negative relation between affective instability of positive emotion (as operationalized by MSSDs) and people's resting heart rate variability, a physiological indicator of emotion regulation capacity. In Koval, Pe, et al. (2013) we disentangle the different dynamics of emotion (variability, instability and inertia) in relation to depressive symptoms. We show that depressive symptomatology is independently characterized by higher inertia and variability in negative emotion, but not instability. In Pe, Koval, \& Kuppens (2013) we show how affective updating ability (assessed with an emotional n-back task) is related to higher levels of life satisfaction in daily life.

${ }^{9}$ Analyses involving ESM data are based on $n=95$; CES-D descriptive statistics are calculated from $n=99$.
} 
Upon giving their informed consent, participants once again completed the CES-D in an initial lab session, together with the DASS-21 (Lovibond \& Lovibond, 1995) and several other self-report questionnaires, irrelevant for this report. Next, they participated in an ESM study, in which they reported their momentary experiences of positive and negative emotion multiple times a day for the duration of one week.

Depressive symptoms. The CES-D was used to assess participants' levels of depressive symptomatology. This 20 -item questionnaire lets respondents rate how frequently they have experienced a range of depressive symptoms (e.g. I felt depressed) in the past week, ranging from 0 (rarely or none of the time) to 3 (most or all of the time). Internal consistency was high $(\alpha=.92)$ and the scale exhibited a high correlation with the CES-D completed at prescreening ${ }^{10}, r(97)=.72, p<.001$. A substantial amount of participants $(n=36)$ reported depressive symptom scores exceeding the clinical cut-off (i.e. CES-D $\geq 16$ ) proposed by Radloff (1977). Descriptive statistics for the CES-D are displayed in Table 1.

Anxiety and stress-related symptoms. We assessed participants' anxiety and distress levels with the DASS-21 questionnaire (Lovibond \& Lovibond, 1995) ${ }^{11}$. This instrument consists of three 7-item subscales, aiming to differentiate between typical symptoms of depression $^{12}$ (i.e. absence of positive affect and loss of incentive; e.g. I couldn't seem to experience any positive feelings at all), anxiety (i.e. fearfulness and autonomic arousal; e.g. I felt I was close to panic) and stress (i.e. high negative affect or experiencing distress; e.g. I tended to over-react to situations; Brown, Chorpita, Korotitsch, \& Barlow, 1997). Participants were asked to indicate how frequently they had experienced each item over the last week on a

\footnotetext{
${ }^{10}$ Results presented below are all based on the CES-D scores measured at the time of the study, not prescreening.

${ }^{11}$ Note that only 87 out of 95 participants completed the DASS-21 survey, as this questionnaire did not belong to the compulsory part of our baseline session. They could complete this questionnaire while waiting for other participants to finish.

12 Participants' depressive symptom scores in the results presented below are all based on the CES-D, not the DASS-21 depression subscale. Nevertheless, replicating our analyses with the DASS-21 depression subscale yields highly similar results and supports identical conclusions.
} 
scale ranging from 0 (never) to 3 (almost always). All subscales showed high internal consistency (depression $\alpha=.90$, anxiety $\alpha=.85$, stress $\alpha=.86$ ). Descriptive statistics for all subscales and their intercorrelations are presented in Table 1.

ESM protocol. The relationship between momentary positive and negative affect was assessed in daily life using experiencing sampling. After providing participants with a Palm Tungsten E2 palmtop, we instructed them to rate their current experience of positive (relaxed, happy) and negative (sad, depressed, anxious, anger, stressed) emotions on a continuous scale, ranging from 1 (not at all) to 100 (very much). The selection of our affect items was intended to cover each of the four quadrants of the affective circumplex proposed by Russell (1980; PA: relaxed $=$ low arousal, happy $=$ high arousal; NA: sad and depressed $=$ low arousal, anxious, anger and stressed = high arousal), which was confirmed by the Dutchlanguage valence and arousal norms for these items created by Moors, Houwer, Hermans, Winne and Brysbaert (2013). Palmtops were programmed to beep ten times a day for seven consecutive days. Using a stratified random interval scheme - with beeps occurring randomly within equal intervals during waking hours (10 AM-10 PM) -, participants, on average, received a beep every $73.30 \mathrm{~min}(S D=29.33)$. Compliance was high, with participants completing an average of $91.5 \%$ of the momentary emotional assessments $(S D=6.2 \%)$. To create a measure for positive and negative affect, ratings of emotions with the same valence were averaged at each beep. Reliability estimates were calculated according to Nezlek (2016) and showed acceptable to excellent internal consistency for NA $(\alpha$ measurement occasion $=.63 ; \alpha$ person level $=.98)$ and PA $(\alpha$ measurement occasion $=.65 ; \alpha$ person level $=.97)$. Descriptive statistics for PA, NA and their relationship are presented in Table 1. 


\section{THE BIPOLARITY OF AFFECT AND DEPRESSIVE SYMPTOMS}

Table 1. Means, standard deviations and between-person correlations among all measures in Study 1.

\begin{tabular}{|c|c|c|c|c|c|c|c|c|c|}
\hline & \multirow[b]{2}{*}{ Mean $(S D)$} & \multicolumn{8}{|c|}{ Correlations } \\
\hline & & 1 & 2 & 3 & 4 & 5 & 6 & 7 & 8 \\
\hline 1. CES-D & $14.67(9.67)$ & & & & & & & & \\
\hline 2. DASS-21 depression & $4.85(5.12)$ & $.76^{* * *}$ & & & & & & & \\
\hline 3. DASS-21 anxiety & $4.58(4.53)$ & $.62 * * *$ & $.66^{* * *}$ & & & & & & \\
\hline 4. DASS-21 stress & $7.12(4.82)$ & $.62 * * *$ & $.76^{* * *}$ & $.65 * * *$ & & & & & \\
\hline 5. Mean momentary PA & $57.26(13.19)$ & $-.60 * * *$ & $-.64 * * *$ & $-.50 * * *$ & $-.55^{* * * *}$ & & & & \\
\hline 6. Mean momentary NA & $17.66(11.03)$ & $.61 * * *$ & $.67 * * *$ & $.53 * * *$ & $.68 * * *$ & $-.63 * * *$ & & & \\
\hline 7. SD momentary PA & $17.32(4.54)$ & $.23^{*}$ & .15 & $.19^{\dagger}$ & $.25^{*}$ & $-.27 * *$ & $.30 * *$ & & \\
\hline 8. SD momentary NA & $10.16(4.50)$ & $.55^{* * * *}$ & $.50 * * *$ & $.39 * * *$ & $.48 * * *$ & $-.51 * * *$ & $.78^{* * * *}$ & $.53 * *$ & \\
\hline 9. PA-NA correlation & $-.50(0.22)$ & $-.34 * * *$ & $-.21^{\dagger}$ & -.17 & -.17 & .13 & $-.38 * * *$ & $-.36^{* * *}$ & $-49 * * *$ \\
\hline
\end{tabular}

Note. CES-D and DASS-21 scores were obtained at baseline; mean and SD measures of momentary PA and NA refer respectively to an individual's average experience of and variability in these affective states during the ESM protocol. ${ }^{\dagger} p<.100 * p<.050 . * * p<.010 . * * * p<.001$. 


\section{Results and Discussion}

\section{Relation between affective bipolarity and depressive symptoms. As Figure 1}

illustrates, considerable variation was observed in the relationship people display between momentary positive and negative affect (range $=-.82-.12$ ), with some participants exhibiting a nearly perfect bipolar relationship (i.e. correlation close to -1.0$)^{13}$, while other people's affective states rather showed independence (i.e. correlation close to .0) ${ }^{14}$.

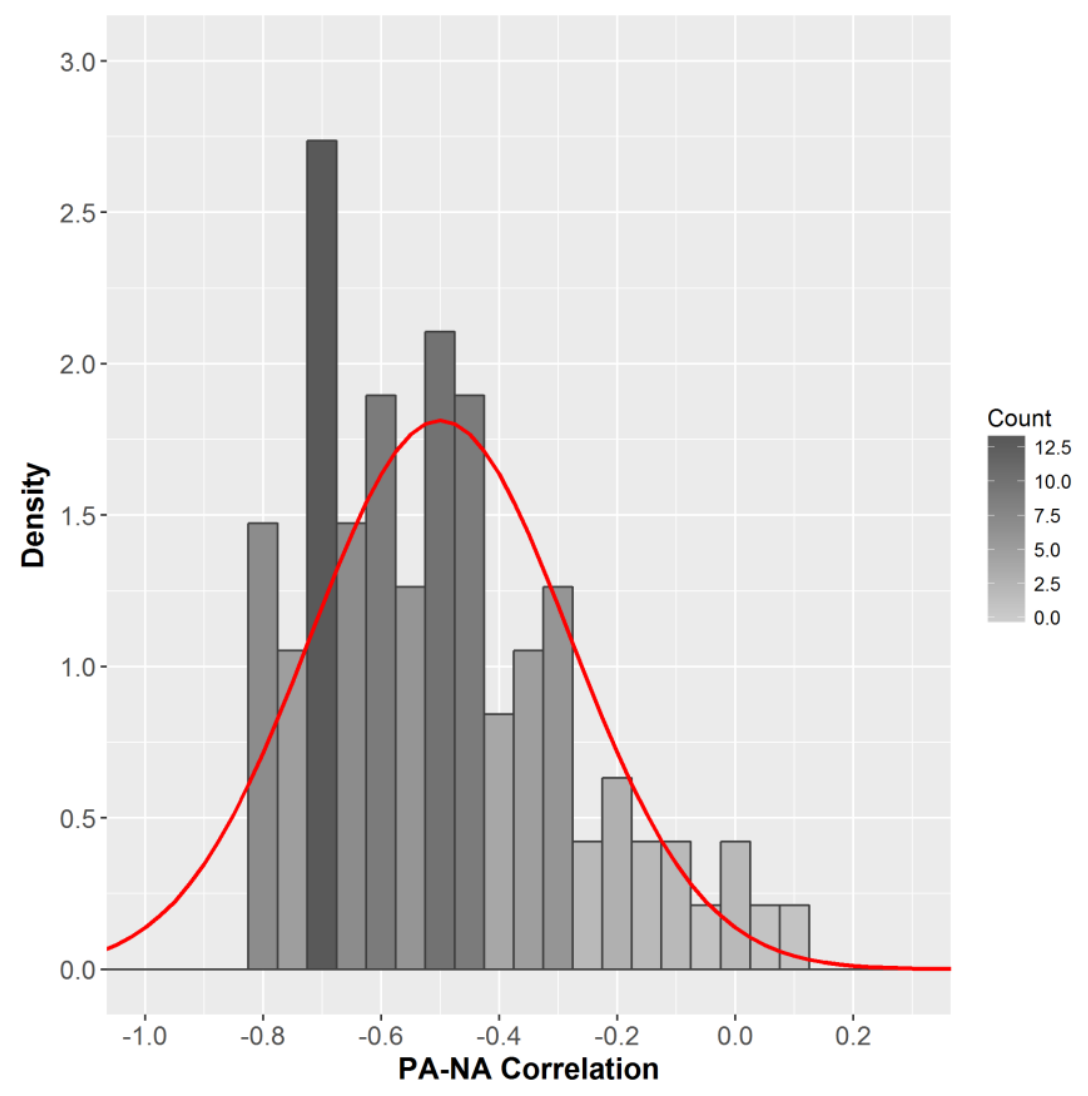

Fig. 1. Density and frequency distribution of participants' PA-NA correlation $(n=95)$, showing substantive individual differences. This histogram is accompanied by its empirically inferred normal distribution.

\footnotetext{
${ }^{13}$ Note that measurement error generally attenuates correlation coefficients, masking a pure bipolar relationship between positive and negative affect (Green et al., 1993).

${ }^{14}$ Some participants $(n=4)$ even displayed a slight positive relation between their momentary PA and NA. While all presented analyses are based on the total sample, excluding these positive cases yielded similar results in all reported studies.
} 
Pearson correlations among all measures are displayed in Table 1. The PA-NA correlation showed a significant negative relationship with participants' depressive symptom severity: individuals who reported more depressive complaints showed a stronger inverse correlation between momentary positive and negative affect ${ }^{15}$. That is to say, people who encounter higher levels of depressive symptoms tend to experience PA and NA more as two opposite poles of a single dimension, while people with lower levels experience positive and negative affect more independently ${ }^{16}$.

Controlling for average levels and variability in PA and NA. Using hierarchical multiple regression, we next examined whether this stronger bipolarity in people who experience high levels of depressive symptoms remained after controlling for mean levels of PA and NA. Participants' CES-D score was our key dependent variable in both models. In a first step (Model 1), we introduced mean levels of PA and NA. In a second step (Model 2), the PA-NA correlation was included as an additional predictor. Table 2 presents the results of these analyses. Both mean levels of PA and NA predicted depressive symptom severity in the expected direction. Adding the PA-NA correlation to this prediction led to a meaningful increase in explained variance, suggesting this affective relationship has unique explanatory power above and beyond mean levels of PA and NA $(\beta=-.17, p=.041)$.

Next, to exclude the possibility our results were merely driven by the observed variability in these affective states, we additionally included the standard deviations of participants' momentary PA and NA in the prediction of their CES-D score in a final model

\footnotetext{
${ }^{15}$ With the DASS-21 depression subscale this relation was only marginally significant $(p=.054)$. Note, however, that our sample with the DASS-21 measures was slightly smaller $(n=87)$, leading to a decrease in statistical power.

${ }^{16}$ In all reported studies we additionally computed the non-parametric Spearman rank PA-NA correlations and related these to participants' CES-D scores, as Pearson correlations are known to be sensitive to the distribution of its variables, with especially negative affect being likely to violate assumptions of normality. In all reported studies both types of correlations produced highly identical results, convincing us the present results are not driven by skewed distributions in PA and NA.
} 
(Model 3). This led the PA-NA correlation to become marginally significant $(\beta=-.16, p=$ $.080)$.

Table 2. Hierarchical multiple regression on CES-D scores, exploring the unique main effect of the PA-NA correlation in Study 1.

\begin{tabular}{|c|c|c|c|c|c|c|}
\hline & $R^{2}(\operatorname{adj})$ & $\Delta R^{2}$ & $\Delta F$ & $\beta$ & $T$ & $p$ \\
\hline Model 1 & .44 & .45 & $37.89 * * *$ & & & \\
\hline Intercept & & & & & 4.51 & $<.001$ \\
\hline Mean momentary PA & & & & -.36 & -3.66 & $<.001$ \\
\hline Mean momentary NA & & & & .38 & 3.85 & $<.001$ \\
\hline Model 2 & .46 & .03 & $4.31 *$ & & & \\
\hline Intercept & & & & & 4.36 & $<.001$ \\
\hline Mean momentary PA & & & & -.40 & -4.01 & $<.001$ \\
\hline Mean momentary NA & & & & .30 & 2.79 & .006 \\
\hline PA-NA correlation & & & & -.17 & -2.08 & .041 \\
\hline Model 3 & .46 & .01 & 0.50 & & & \\
\hline Intercept & & & & & 3.95 & $<.001$ \\
\hline Mean momentary PA & & & & -.40 & -3.97 & $<.001$ \\
\hline Mean momentary NA & & & & .20 & 1.41 & .164 \\
\hline SD momentary PA & & & & -.08 & -.83 & .408 \\
\hline SD momentary NA & & & & .16 & 1.12 & .267 \\
\hline PA-NA correlation & & & & -.16 & -1.77 & .080 \\
\hline
\end{tabular}

Note. ${ }^{*} p<.050 . * * p<.010 . * * * p<.001$.

Impact of PA and NA construct operationalization. Because the relation between positive and negative affect may vary as a function of how these constructs are operationalized (Barrett \& Russell, 1998), we next examined to what extent the relation between affective bipolarity and depressive symptoms changed as function of using particular 
item combinations to construct PA and NA composites. By doing so, we checked whether our findings are robust across different compositions of our affective constructs. In a multiverse analysis (Steegen, Tuerlinckx, Gelman, \& Vanpaemel, 2016) we computed every PA-NA correlation by making all possible combinations from the two positive and five negative emotion items. This resulted in a set of 93 possible correlations, which we then each related to participants' CES-D scores.
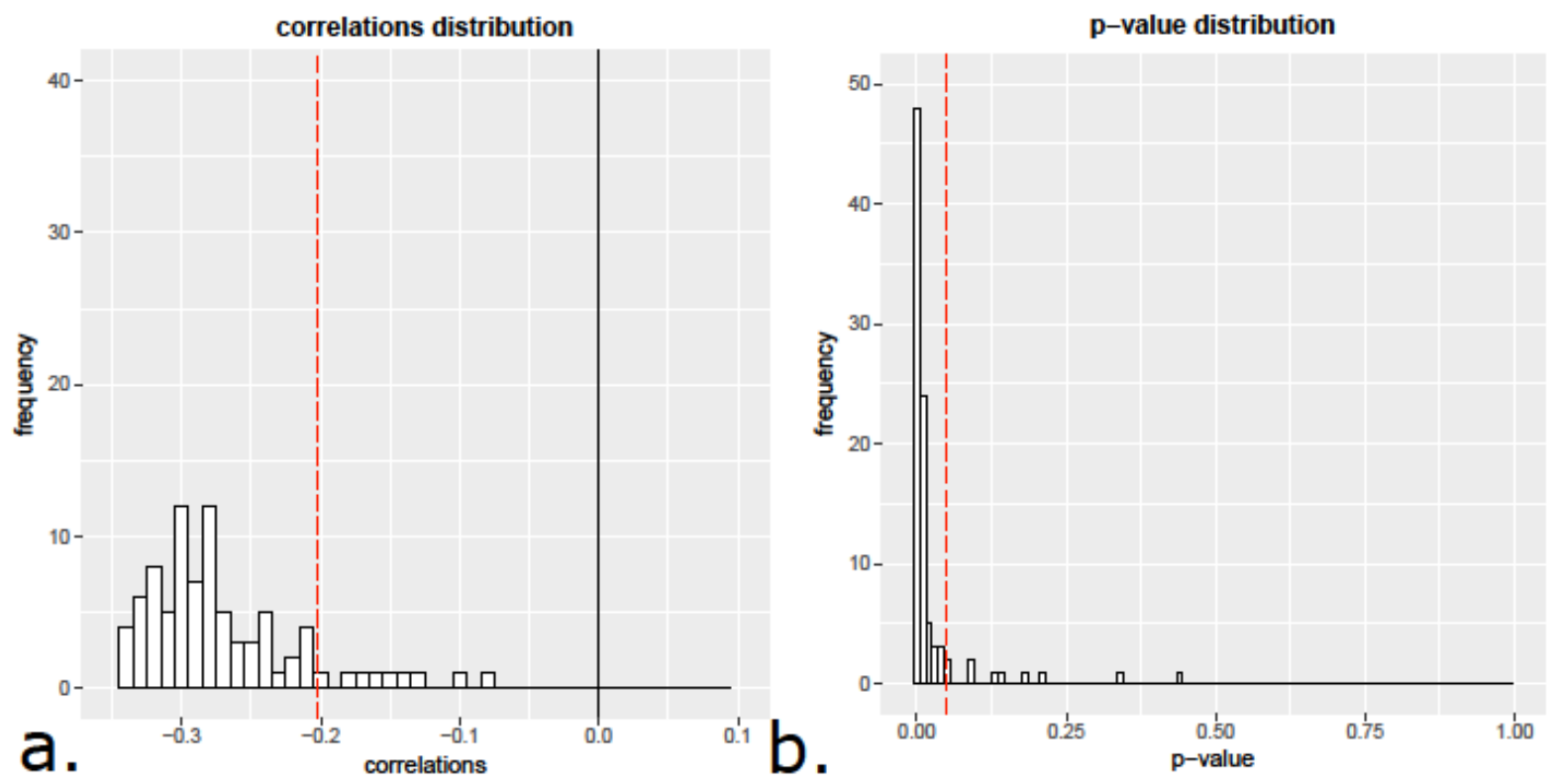

Fig. 2. Distribution of the observed relations between participants' CES-D scores and all possible PANA correlations $(n=93)$, accompanied with the distribution of corresponding $p$-values. Red dashed lines indicate the significance threshold.

As can be seen from Figure 2, the large majority of possible PA-NA correlations (each based on a different combination of affect items) showed a negative and significant association with depressive symptom severity. Only in eight cases was affective bipolarity not related to participants' CES-D scores, convincingly illustrating our findings are not specific to a particular combination of positive and negative states. Non-significant combinations are listed in Supplementary Materials (Table 4).

Specificity to depressive symptoms. To investigate to what degree affective bipolarity is specific for depressive symptomatology, we next estimated a series of multiple 
regressions to examine the unique main effect of depressive symptoms when controlling for anxiety and stress complaints. To assess participants' anxiety and distress symptoms, we used the corresponding DASS-21 subscales. To create a measure for depressive symptoms, the average of participants' (standardized) CES-D and DASS-21 depression subscale scores was used $^{17}$. Results of these analyses are presented in Table 3.

Table 3. Multiple regression models exploring the unique main effects of stress, anxiety and depressive symptoms in the prediction of participants' PA-NA correlation in Study 1.

$\beta \quad t \quad p$

\begin{tabular}{lllll}
\hline Model 1 & -.31 & -3.11 & .002 \\
Depressive symptoms & & & & \\
& Model 2 & -.29 & -2.04 & .045 \\
Depressive symptoms & & & & \\
Anxiety symptoms & .03 & 0.23 & .817
\end{tabular}

\section{Model 3}

Depressive symptoms

$-.32-2.05$

.043

Stress symptoms

.07

0.44

.663

\section{Model 4}

Depressive symptoms $\begin{array}{lll}-.33 & -1.92 & .058\end{array}$

Anxiety symptoms

$\begin{array}{lll}.02 & 0.11 & .914\end{array}$

Stress symptoms

$.06 \quad 0.38$

.703

Note. Participants' depressive symptom scores were based on the average of their (standardized) scores on the CES-D and DASS-21 depression subscale. For the anxiety and stress symptoms the original DASS-21 subscales were used.

17 As the CES-D was used throughout our manuscript to assess participants' depressive symptomatology, but the DASS-21 has its own depression subscale, we combined both measures for our specificity analyses. 
Our combined depressive symptom measure showed a negative relation with participants' PA-NA correlation (i.e. affective bipolarity; Model 1). Adding symptoms of anxiety (Model 2) or stress (Model 3) did not change this relation and these symptoms themselves did not meaningfully contribute in the prediction of affective bipolarity. This suggests affective bipolarity is uniquely related to depressive symptoms and is not attributable to possible overlap with anxiety or psychological distress in general. In Model 4 we included all symptom types together. This led depressive symptom severity to become marginally significant $(\beta=-.33, p=.058)$. Again, stress and anxiety-related symptoms showed no association with affective bipolarity ${ }^{18}$.

To summarize, Study 1 confirms that considerable variability across individuals exists in the way positive and negative affect are related in human experience. While for most people PA and NA are moderately (negatively) correlated, some show a more independent relationship, while still for others these affective states can be seen as extreme opposites of a single dimension. Central to our question, our results indicate this relationship is related to individuals' depressive symptom severity: a bipolar experience of momentary positive and negative affect is associated with more depressive symptoms. This relation holds for different types of PA and NA operationalizations and after controlling for average levels of these affective states. Finally, the relation of affective bipolarity appears to be specific to depressive symptoms in comparison to other related dimensions of individual differences in anxiety and distress levels.

\section{Study 2}

Due to the cross-sectional nature of Study 1, making directional claims about the relationship between depressive symptom severity and the PA-NA correlation was not possible. For this reason, we analyzed data from a longitudinal design in Study 2. Here, we

\footnotetext{
${ }^{18}$ Although substantial intercorrelations between depressive, anxiety and stress complaints were observed, Variance Inflation Factors (VIF) of all predictors did not indicate multicollinearity (all VIFs $\leq 2.61$ ).
} 
assessed participants' depressive symptoms and PA-NA correlation multiple times over a 1year course, which enabled us to determine whether the bipolarity of affect follows from, or rather serves as a predictor of these symptoms.

\section{Participants and Prescreening}

Two hundred KU Leuven bachelor students were recruited to participate as part of a larger longitudinal study on emotional functioning and psychological well- being ${ }^{19}$. The study consisted of three measurement waves in which participants took part in an experience sampling protocol for seven days. Wave 1 took place at the beginning of the academic year, while Wave 2 and 3 followed respectively four months and one year later. Sample size was determined to ensure sufficient power to detect small to medium effects $(r=.30, \alpha=.05)$, allowing for $25 \%$ attrition over the 1-year study. Participants received $€ 60$ for their participation in each wave, with a $€ 60$ bonus for completing all three waves.

To guarantee our sample again included participants with a broad and balanced range of depressive symptoms, a similar sampling protocol was used as reported in Study 1. From an initial pool of 686 students (235 men) commencing their first year of higher education, we selected 180 participants with varying depressive symptom scores as measured with the CES$\mathrm{D}($ range $=0-39, M=14.41, S D=8.41, \alpha=.90)$. In order to achieve our desired sample size, an additional group of 22 participants enrolled after the study had already begun and therefore did not complete CES-D prescreening ${ }^{20}$.

\footnotetext{
19 These data have been previously used to test other hypotheses, but there is no overlap between previous work and the current research. In Brose, Wichers, \& Kuppens (2017) we disentangle the relationship between stressful events in daily life and depressive symptoms. We show how stressful experiences precede, but not follow from depressive symptoms. In Koval et al. (2015) we show how higher inertia in negative emotion is related to decreased negative affect recovery following negative stimuli, both in the lab and in daily life. In Pe et al. (2015) we show how affective updating ability (assessed with an emotional n-back task) is related to greater emotion reactivity and facilitates quicker recovery from watching negative film clips in the lab. In Pe et al. (2016) we show how affective updating ability (assessed with an emotional n-back task) predicts the experience of depressive symptoms 4 months and 1 year later, but only when people experience high levels of stress.

20 The CES-D was administered again before the start of the study and participants who did not complete the CES-D prescreening did not significantly differ from those who did in their CES-D scores $(p=.695)$.
} 
For Wave 1, two participants showed poor compliance with the ESM protocol (i.e., < $50 \%$ response rate), which led to an initial sample of 200 participants (90 men; $M_{\text {age }}=18.32$, $\left.S D_{\text {age }}=0.97\right)$. By the start of Wave 2, ten people (i.e. 5\%) had dropped out, (190 participants; 84 men; $M_{\text {age }}=18.64, S D_{\text {age }}=1.03$ ), followed by another 13 participants (i.e. $7 \%$ ) for Wave 3 (177 participants; 79 men; $\left.M_{\text {age }}=19.28, S D_{\text {age }}=1.00\right)$.

\section{Materials and Procedure}

After providing informed consent, we asked participants prior to each measurement wave to complete a battery of baseline questionnaires, including the CES-D and the DASS21. Subsequently, participants' momentary positive and negative affect was assessed several times per day for one week in an ESM protocol.

Depressive symptoms. Preceding each ESM protocol, the CES-D was administered once again to assess participants' current depressive symptomatology. Depressive symptom scores showed high internal consistency for all measurement waves (Wave 1: $\alpha=.90$; Wave 2: $\alpha=.92 ;$ Wave $3: \alpha=.88$ ) and showed moderate cross-temporal stability (see Supplementary Materials, Table 5 for cross-wave correlations). In Wave 1, 65 participants reported depressive symptom scores surpassing Radloff's (1977) clinical threshold (i.e. CES$\mathrm{D} \geq 16$ ), followed by 45 and 35 participants in Wave 2 and 3, respectively. CES-D descriptive statistics for each wave are presented in Table 4.

Anxiety and stress-related symptoms. The DASS-21 survey was used to differentiate between participants' depressive, anxiety and distress symptoms, prior to each measurement wave. Each subscale showed good internal consistency in all waves (Wave 1: depression $\alpha=.82$, anxiety $\alpha=.67$, stress $\alpha=.81$; Wave 2: depression $\alpha=.87$, anxiety $\alpha=$ .70 , stress $\alpha=.83$; Wave 3: depression $\alpha=.84$, anxiety $\alpha=.72$, stress $\alpha=.82$ ). Descriptive statistics and intercorrelations among all DASS-21 subscales are displayed in Table 4 for each wave. Cross-wave correlations are presented in Supplementary Materials (Table 5). 
ESM protocol. In all three measurement waves the relationship between momentary positive and negative affect was assessed using experience sampling. Receiving a Motorola Defy plus Smartphone, participants rated their current experienced positive (happy, relaxed, cheerful) and negative (sad, anxious, depressed, anger, stressed) emotions, using a visual slider scale from 0 (not at all) to 100 (very much). Smartphones were programmed to beep ten times a day (10 AM-10 PM) for seven consecutive days. Similar to Study 1, a stratified random interval scheme was adopted, in which participants, on average, received a beep every $71.7 \min (S D=29.2)$ during Wave 1 , every $71.9 \min (S D=29.5)$ during Wave 2 and every $72.0 \min (S D=29.5)$ during Wave 3. Participants responded on average to $87.27 \%(S D=$ $9.05 \%), 87.87 \%(S D=8.98 \%)$ and $88.35 \%(S D=8.69 \%)$ of the programmed beeps at each measurement wave respectively, reflecting good compliance. Again, PA and NA scores were calculated for each beep by averaging participants' ratings for each valence. Internal consistency calculated following Nezlek (2016) showed acceptable to excellent reliability for NA (Wave 1: $\alpha$ measurement occasion $=.63 ; \alpha$ person level $=.98 ;$ Wave $2: \alpha$ measurement occasion $=.65 ; \alpha$ person level $=.98 ;$ Wave $3: \alpha$ measurement occasion $=.63 ; \alpha$ person level $=.98)$ and PA (Wave 1: $\alpha$ measurement occasion $=.68 ;$ Wave $2: \alpha$ measurement occasion $=.57 ; \alpha$ person level $=.98 \alpha$ person level $=.97 ;$ Wave $3: \alpha$ measurement occasion $=.55 ; \alpha$ person level $=.97)$. Descriptive statistics for PA, NA and their relationship in each wave are presented in Table 4. 
THE BIPOLARITY OF AFFECT AND DEPRESSIVE SYMPTOMS

Table 4. Means, standard deviations and between-person correlations among all measures in each wave, for Study 2 .

\begin{tabular}{|c|c|c|c|c|c|c|c|c|c|c|c|c|c|c|c|c|c|c|c|c|c|c|c|c|c|c|}
\hline & \multicolumn{8}{|c|}{ Wave 1} & \multicolumn{9}{|c|}{ Wave 2} & \multicolumn{9}{|c|}{ Wave 3} \\
\hline & \multirow[b]{2}{*}{ Mean $(S D)$} & \multicolumn{7}{|c|}{ Correlations } & \multicolumn{9}{|c|}{ Correlations } & \multirow{2}{*}{\multicolumn{3}{|c|}{ Mean $(S D)$}} & \multirow[b]{2}{*}{3} & \multirow[b]{2}{*}{4} & \multirow[b]{2}{*}{5} & \multirow[b]{2}{*}{6} & \multirow[b]{2}{*}{7} & \multirow[b]{2}{*}{8} \\
\hline & & 1 & 2 & 3 & 4 & 5 & 6 & 8 & Mean $(S D)$ & 1 & 2 & 3 & 4 & 5 & 6 & 7 & 8 & & & & & & & & & \\
\hline 1. CES-D & $12.52(7.74)$ & & & & & & & & $11.58(8.90)$ & & & & & & & & & $9.63(7.04)$ & & & & & & & & \\
\hline 2. DASS-21 depression & $3.24(2.97)$ & $.58^{* * * *}$ & & & & & & & $2.95(3.33)$ & $.63^{* * * *}$ & & & & & & & & $2.33(2.59)$ & $.61^{* * *}$ & & & & & & & \\
\hline 3. DASS-21 anxiety & $2.92(2.56)$ & $.30^{* * * *}$ & $.47 * * *$ & & & & & & $2.57(2.47)$ & $.38 * * *$ & $.47 * * *$ & & & & & & & $2.24(2.52)$ & $.33^{* * * *}$ & $.48^{* * *}$ & & & & & & \\
\hline 4. DASS-21 stress & $5.11(3.48)$ & $.51^{* * * *}$ & $.58 * * *$ & $.54 * * *$ & & & & & $4.04(3.45)$ & $.46^{* * *}$ & $.61 * * *$ & $.60^{* * * *}$ & & & & & & $3.85(3.29)$ & $.49 * * *$ & $.54 * * *$ & $.54 * * *$ & & & & & \\
\hline 5. Mean momentary PA & $56.87(9.99)$ & $-.45^{* * *}$ & $*-.35 * * *$ & $-.18^{*}$ & $-.32 * * *$ & & & & $57.38(11.33)$ & $-36 * * *$ & $-.36^{* * * *}$ & $-.20^{* * *}$ & $-.23 * *$ & & & & & $56.55(11.45)$ & $-.38 * * *$ & $* 34 * * *$ & $.18 *$ & $-.25^{* * * *}$ & & & & \\
\hline 6. Mean momentary NA & $14.31(8.44)$ & $.42 * * *$ & $.45 * * *$ & $.38 * * *$ & $.45^{* * * *}$ & $-.43 * * *$ & & & $12.67(8.76)$ & $.42 * * *$ & $.31 * * *$ & $.28^{* * * *}$ & $.43^{* * * *}$ & $-.39 * * *$ & & & & 12.08 & $.35 * * *$ & $.42 * * *$ & $.30 * * *$ & $.42 * * *$ & $-.36 * * *$ & & & \\
\hline 7. SD momentary PA & $16.27(4.68)$ & $.25^{* * * *}$ & $.27^{* * * *}$ & $.14 *$ & $.32 * * *$ & $-.20^{* *}$ & $.28 * * *$ & & $15.16(4.89)$ & $.18^{*}$ & $.13^{\dagger}$ & $.14 *$ & $.16^{*}$ & $-.16^{*}$ & $.19^{*}$ & & & $15.48(5.08)$ & $.19^{* *}$ & $.14^{\dagger}$ & .10 & $.30^{* * * *}$ & $-.22 * *$ & $.23^{* *}$ & & \\
\hline 8. SD momentary NA & $9.60(4.29)$ & $.35^{* * * *}$ & $.40 * * *$ & $.34^{* * * *}$ & $.42^{* * * *}$ & $-.33 * * *$ & $* .70 * * *$ & $.63^{* * *}$ & $8.90(4.74)$ & $.25 * * *$ & $.16^{*}$ & $.15^{*}$ & $.25^{* * *}$ & $-.27 * * *$ & $.63^{* * * *}$ & $.57 * * *$ & & $8.86(4.53)$ & $.30 * * *$ & $.30 * * *$ & $.30 * * *$ & $.48^{* * * *}$ & $-.26 * * *$ & $.64 * * *$ & *. $.61 * * *$ & \\
\hline 9. PA-NA correlation & $-.48(0.22)$ & $-.22 * *$ & $-.21 * *$ & -.03 & $-.19^{* *}$ & .02 & $-.37 * *$ & $-.44 * * *-.52 * * *$ & $-.42(.25)$ & $-.13^{\dagger}$ & $-.17 *$ & -.04 & $-.16^{*}$ & -.03 & $-.15^{*}$ & $-.32 * * *$ & $*-.32 * * *$ & $-.44(0.24)$ & $-.25^{* * * *}$ & $k^{*}-21 * *$ & -.04 & $-.25 * * *$ & .02 & $-.13^{\dagger}$ & $-.39 * * *$ & $-.35^{* * *}$ \\
\hline
\end{tabular}

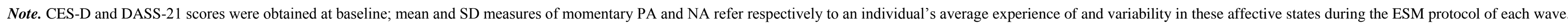
${ }^{\dagger} p<.100 * p<.050 . * * p<.010 . * * * p<.001$. 
THE BIPOLARITY OF AFFECT AND DEPRESSIVE SYMPTOMS
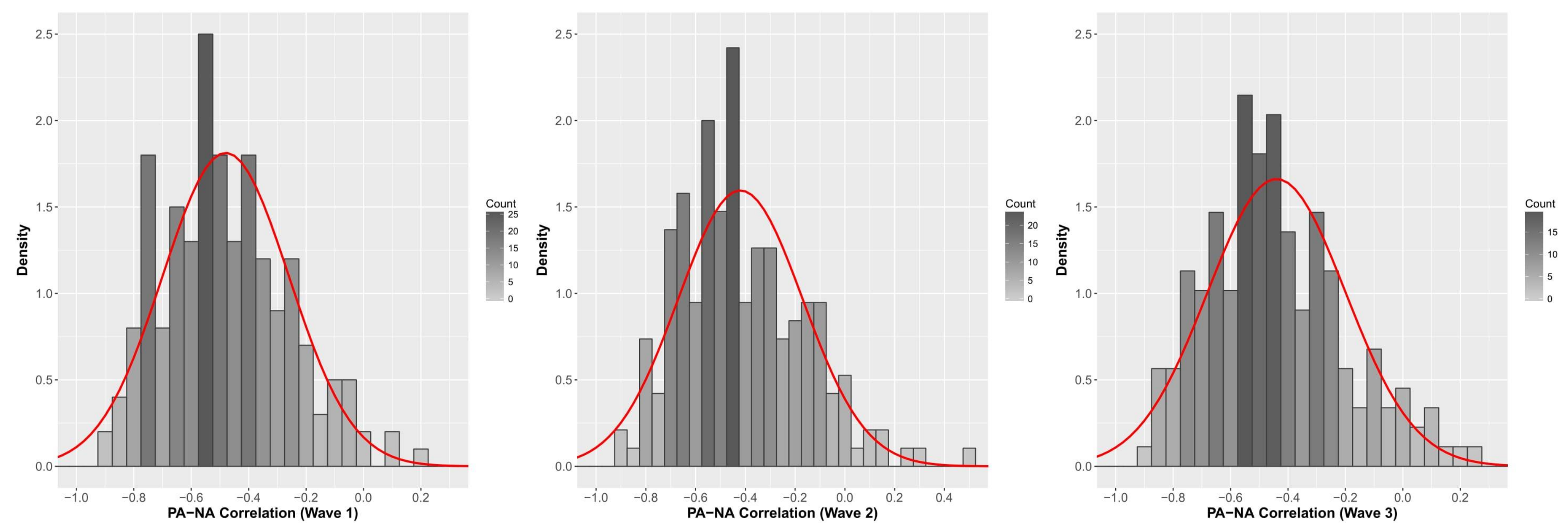

Fig. 3. Density and frequency distributions of participants' PA-NA correlation for Wave $1(n=200)$, Wave $2(n=190)$ and Wave $3(n=177)$. Each histogram is accompanied by its empirically inferred normal distribution 


\section{Results and Discussion}

Relation between affective bipolarity and depressive symptoms within waves. As displayed in Figure 3 and similarly to Study 1, we observed substantial individual differences in the negative relationship between momentary positive and negative affect in all waves (Wave 1: range $=-.90-.21 ;$ Wave 2: range $=-.91-.48 ;$ Wave 3: range $=-.88-.24)$.

Furthermore, the PA-NA relationship showed moderate cross-temporal stability over a 1-year course (see Supplementary Materials, Table 5).

Pearson correlations among all measures in each wave are presented in Table 4. For both Wave 1 and 3, the PA-NA relationship was negatively associated with participants' CES-D scores, replicating our findings of Study 1 . For Wave 2, only a marginally significant negative correlation was observed $(p=.069)^{21}$. As participants reported more depressive symptoms, they displayed stronger bipolarity of affect.

\section{Directionality between affective bipolarity and depressive symptoms across}

waves. To determine temporal directionality between participants' depressive symptom severity and PA-NA correlations, vector autoregression was used (VAR; Hamilton, 1994). In addition to the autoregressive effect of the outcome, these models investigate the prospective predictive value of other variables. In Model 1, CES-D scores at Wave 2 were predicted by PA-NA correlations and CES-D scores at Wave 1 (see Supplementary Materials, Table 6). In Models 2 and 3, CES-D scores at Wave 3 were predicted by PA-NA correlations and CES-D scores at Wave 1 and 2, respectively. In Model 4, PA-NA correlation at Wave 2 were predicted by PA-NA correlations and CES-D scores at Wave 1. Finally, in Models 5 and 6, PA-NA correlations at Wave 3 were predicted by PA-NA correlations and CES-D scores at Wave 1 and 2, respectively. In all models, we also controlled for mean levels of PA and NA.

\footnotetext{
21 Using the DASS-21 depression subscale, however, did produce a significant relation $(p=.017)$.
} 
To reduce the familywise error rate, we used an alpha Bonferroni-corrected for the number of models tested $(\alpha=0.05 / 6)$.

Wave 1

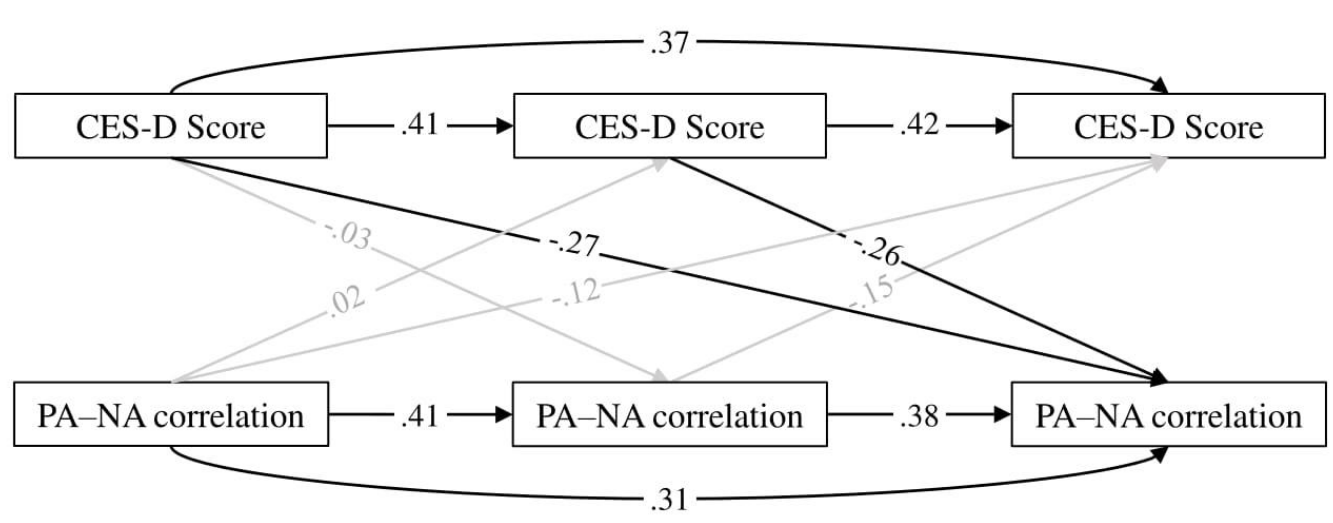

Fig. 4. VAR models visualized to determine directionality between CES-D scores and PA-NA correlations across the three measurement waves. The figures represent the coefficients of each crossregressive effect after controlling for mean PA and NA. Black lines indicate significance, gray lines indicate non-significance after Bonferroni correction $(\alpha=0.05 / 6)$.

As Figure 4 illustrates, CES-D scores at both Waves 1 and 2 significantly predicted a stronger inverse PA-NA correlation at Wave 3, even after including mean PA and NA and PA-NA correlations at Waves 1 and 2, respectively (Models 5 and 6). This finding, however, did not replicate in Model 4. In contrast, PA-NA correlations at Waves 1 and 2 did not predict any meaningful change in CES-D scores at Waves 2 or 3 (Models 1, 2 and 3). Together, these results are suggestive that affective bipolarity follows from elevated depressive symptoms, rather than being a predictor of depressive symptomatology.

Finally, besides controlling for average levels of PA and NA, we reran all models, additionally controlling for participants' standard deviations of momentary PA and NA (again controlling for multiple testing, $\alpha=0.05 / 6$ ). Concurrently, this affected the predictive value of the PA-NA correlation for Wave $1(\beta=-.11, p=.152)$, but not Wave $3(\beta=-.21, p=.006)$. The predictive capacity of participants' PA-NA correlation in Wave 2 remained nonsignificant $(\beta=-.09, p=.187)$. Longitudinally, conclusions remained identical: higher CES-D 
scores at Wave 1 and 2 predicted stronger inverse PA-NA correlations at Wave 3 (Model 5: $\beta$ $=-.26, \mathrm{p} \leq .001 ;$ Model 6: $\beta=-.26, \mathrm{p} \leq .001$ ), but not from Wave 1 to 2 (Model 4: $\beta=-.03, p$ $=.746$ ). Conversely, in all other models, participants' PA-NA correlation did not predict any meaningful changes in depressive symptoms in the next waves (Model 1: $\beta=.08, p=.316$; Model 2: $\beta=-.12, p=.136$; Model 3: $\beta=-.17, p=.014)$.

Impact of PA and NA construct operationalization. Similarly to Study 1, to investigate to what degree the relation between depressive symptoms and affective bipolarity was a function of how our PA and NA constructs were composed, we conducted a multiverse analysis. From the three positive and five negative emotion items, we computed every possible PA-NA relationship, resulting in a total set of 217 affect correlations. For all waves, these were then related to participants' concurrent CES-D scores.

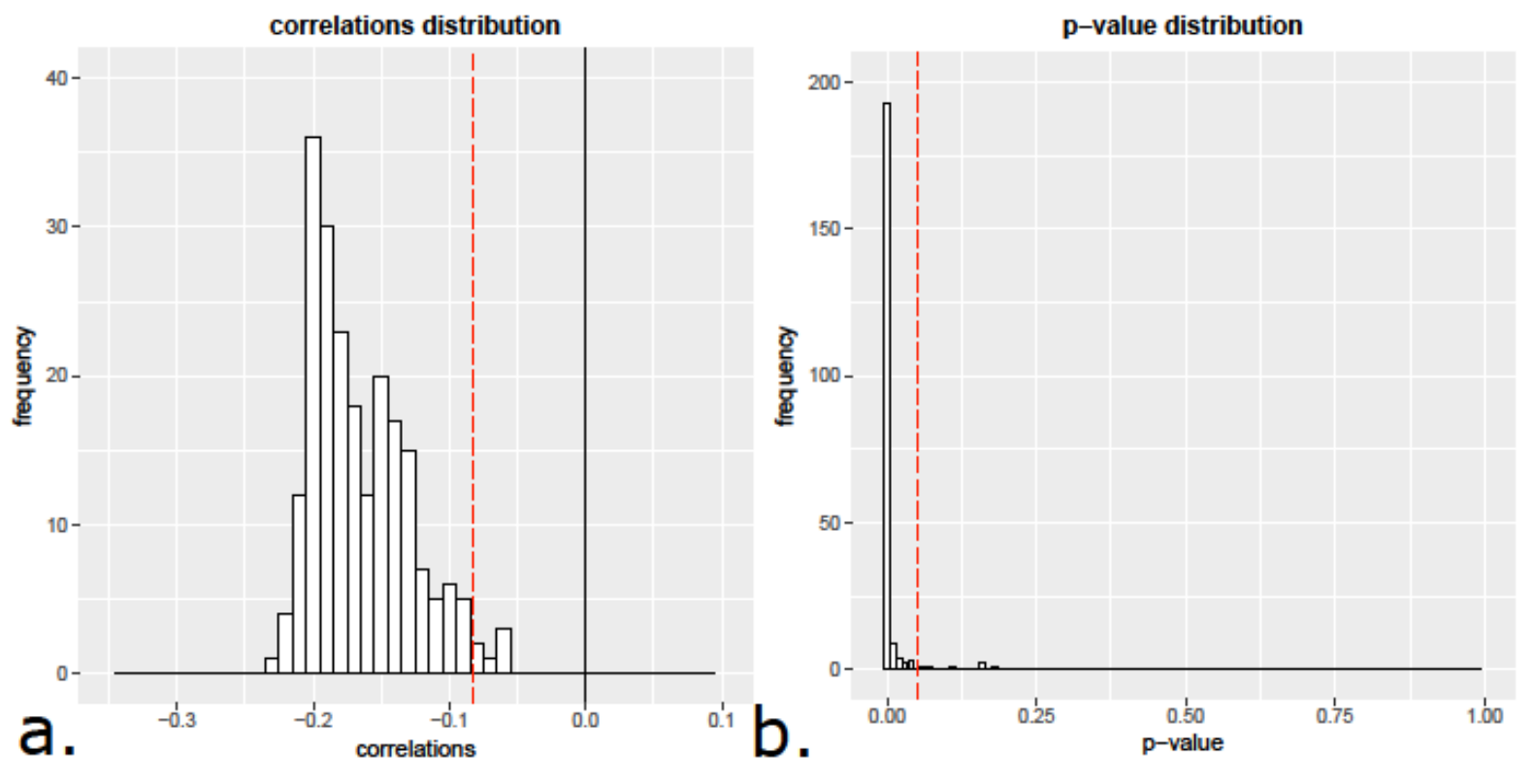

$\overline{\text { Fig. 5. Distribution of the observed relations between participants' CES-D scores and all possible PA- }}$ NA correlations $(\mathrm{n}=217)$ for Study 2 (all waves combined), accompanied with the distribution of corresponding $p$-values. Red dashed lines indicate the significance threshold.

As Figure 5 illustrates, the vast majority of all possible PA-NA correlations (all but six; see Supplementary Materials, Table 4) was meaningfully related with participants' 
concurrent depressive symptom scores, which again indicates robustness of this relation across different compositions of our affective constructs.

Specificity to depressive symptoms. For each wave, we again examined the specificity of this relation in comparison to symptoms of stress and anxiety, in a series of multiple regressions, similarly to Study 1 . Results for all waves are presented in Table 5.

Table 5. Multiple regression models exploring the unique main effects of stress, anxiety and depression symptoms in the prediction of participants' PA-NA correlation in all Waves of Study 2.

\begin{tabular}{|c|c|c|c|c|c|c|c|c|c|}
\hline & \multicolumn{3}{|c|}{ Wave 1} & \multicolumn{3}{|c|}{ Wave 2} & \multicolumn{3}{|c|}{ Wave 3} \\
\hline & $\beta$ & $t$ & $p$ & $\beta$ & $t$ & $p$ & $\beta$ & $t$ & $p$ \\
\hline \multicolumn{10}{|l|}{ Model 1} \\
\hline Depressive symptoms & -.24 & -3.47 & .001 & -.169 & -2.36 & .020 & -.26 & -3.50 & .001 \\
\hline \multicolumn{10}{|l|}{ Model 2} \\
\hline Depressive symptoms & -.28 & -3.65 & $<.001$ & -.20 & -2.39 & .018 & -.30 & -3.67 & $<.001$ \\
\hline Anxiety symptoms & -.09 & 1.20 & .232 & .06 & 0.70 & .483 & .10 & 1.19 & .234 \\
\hline \multicolumn{10}{|l|}{ Model 3} \\
\hline Depressive symptoms & -.20 & -2.27 & .025 & -.12 & -1.30 & .196 & -.17 & -1.94 & .054 \\
\hline Stress symptoms & -.07 & -0.78 & .439 & -.09 & -0.98 & .328 & -.15 & -1.65 & .100 \\
\hline \multicolumn{10}{|l|}{ Model 4} \\
\hline Depressive symptoms & -.22 & -2.50 & .013 & -.14 & -1.51 & .133 & -.21 & -2.34 & .021 \\
\hline Anxiety symptoms & -.13 & 1.62 & .106 & .12 & 1.29 & .199 & .18 & 2.02 & .045 \\
\hline Stress symptoms & -.13 & -1.34 & .182 & -.15 & -1.46 & .146 & -.22 & -2.32 & .021 \\
\hline
\end{tabular}

Note. Participants' depressive symptom scores were based on the average of their (standardized) scores on the CES-D and DASS-21 depression subscale. For the anxiety and stress symptoms the original DASS-21 subscales were used. 
In each wave, bipolarity of affect was meaningfully related with our depressive symptom measure (Model 1). Similarly to Study 1, controlling for participants' anxiety levels did not influence this relation, and these symptoms never showed a significant association with participants' PA-NA correlation themselves (Model 2), which further validates our predictions based on the tripartite model. Unlike Study 1, taking into account stress-related symptoms was less unequivocal across waves (Model 3). While stress complaints were never meaningfully related to participants' PA-NA correlation, controlling for these symptoms occasionally did influence the relation with their depressive symptom scores (i.e. significant in Wave 1, non-significant in Wave 2, marginally significant in Wave 3), suggesting that it is partly (however not uniquely) the distress involved in the experience of depressive symptoms being responsible for the relation with affective bipolarity, which is in line with our predictions based on the DMA (Zautra et al., 1997). Finally, including all symptom types together (Model 4), produced similar results ${ }^{22}$. In Wave 1, bipolarity of affect was only predicted by depressive symptoms, not by participants' anxiety or stress-related complaints. In Wave 2, no relation survived the significant threshold. In Wave 3, depressive symptomatology and distress symptoms predicted affective bipolarity equally ${ }^{23}$. Together, these results suggest that affective bipolarity shows specificity to depressive, and to some degree, distress-related symptoms, but not to anxiety-related symptoms.

In sum, Study 2 largely replicates our concurrent findings of Study 1. Experiencing momentary positive and negative affect in a more bipolar way was associated with higher depressive symptom severity in all waves (although only marginally significant in Wave 2). Again, this relation was robust across various PA and NA operationalizations and showed

\footnotetext{
${ }^{22}$ In each wave, VIFs of all predictors indicated no multicollinearity (Wave $1 \leq 1.88$; Wave $2 \leq 1.94$; Wave $3 \leq$ $1.75)$.

23 Interestingly, anxiety symptoms here meaningfully predict affective independence, which is in line with what we would expect based the tripartite model.
} 
specificity towards depressive (and to some degree distress-related) rather than anxiety symptoms. In addition, this study also extended the conclusions of Study 1 with directional analyses. Looking across waves, results suggest depressive symptomology predicts affective bipolarity up to one year later, rather than the other way around. These cross-wave relations held after controlling for average levels of PA and NA in each wave, indicating affective bipolarity is not merely a function of altered mean levels of these affective states.

\section{Study 3}

In a final study, we aimed to replicate our main hypotheses using a slightly different study design. First, this study made use of a daily diary approach (instead of ESM), allowing to test the focal relation between bipolarity of affect and depressive symptoms on a different time scale in comparison to both previous studies. The time frame in which PA and NA are assessed is known to impact conclusions about their reciprocal relationship (Diener \& Emmons, 1985; Watson, 1988), which may in turn have repercussions for the observed relation between bipolarity of affect and depressive symptoms. Observing how affective bipolarity and depressive symptoms are meaningfully related when PA and NA are assessed momentarily, may thus not necessarily generalize to longer time scales (e.g. a daily average). Second, we used depression's two key symptoms, sadness and anhedonia, as specific manifestations of high NA and low PA, respectively. These symptoms are not regarded as equivalent stand-ins for positive and negative affect, but they are highly conceptually related (Watson \& Clark, 1991; Watson \& Kendall, 1989), leading us to hypothesize that as individuals are characterized with increasingly severe levels of depressive symptomatology, both their depressed mood and anhedonia will be more strongly and positively correlated across time ${ }^{24}$.

\section{Participants and Prescreening}

\footnotetext{
24 Note that, in order to reflect low positive affect, anhedonia should be inversely interpreted, leading to a change in the sign of the correlation.
} 
Participants were recruited to take part in a one-month daily diary study investigating the dynamics of depressive symptoms ${ }^{25}$. They were reimbursed $\$ 1$ for completing a survey each day, receiving an additional $\$ 20$ at the end of the study if they had completed 25 days or more. In order to observe small to medium effects $(r=.30, \alpha=.05)$, we aimed to recruit a sample of 100 participants who currently experienced elevated depressive symptoms.

To enroll participants in our study, we adopted Amazon's Mechanical Turk services (MTurk). The MTurk community was a particularly suitable subject pool for the current study, as it has recently been shown that MTurkers experience more depressive symptoms than regular nonclinical samples (Arditte, Çek, Shaw, \& Timpano, 2015), but at the same time resemble the general population in other demographics more closely than traditional convenience samples (e.g. undergraduates; Casler, Bickel, \& Hackett, 2013).

The Patient Health Questionnaire (PHQ-9; Kroenke, Spitzer, \& Williams, 2001) was used as a prescreening instrument for depressive symptomatology. An initial group of 987 MTurkers (553 men) completed this survey (PHQ-9 range $=0-27, M=6.32, S D=5.90) .207$ individuals had depressive symptom scores exceeding the clinical cut-off (PHQ $\geq 10$; Kroenke et al., 2001). Of this group, 194 people were randomly contacted to participate in our daily diary protocol, expecting about 120 individuals to respond to our request (taking into account an estimated $15 \%$ attrition for a final sample of 100 participants). 121 people (64 men) replied and began our study (PHQ-9 range $=11-27, M=14.48, S D=4.10)$.

Nine participants ${ }^{26}$ were excluded from analyses due to poor compliance with the daily dairy protocol (i.e. $<50 \%$ response rate), leaving a final sample of 112 participants (58 men; $\left.M_{\text {age }}=34.27, S D_{\text {age }}=9.78\right)$.

\footnotetext{
25 These data have been previously used in Dejonckheere, Bastian, Fried, Murphy, \& Kuppens (2017), but there is no overlap between this work and the current research. In this paper, we investigate the relation between people's perceived social pressure not to feel negative and depressive symptoms. We show how this pressure predicts increases in depressive symptoms, but not vice versa.

26 Their PHQ-scores did not differ significantly $(p=.864)$.
} 


\section{Materials and Procedure}

In an initial survey prior to the daily diary protocol, participants' informed consent was obtained and among several other baseline questionnaires, the CES-D was again administered to assess current depressive symptomatology. Next, participants were instructed to rate their depressive symptoms on a daily basis for one month by completing an online dairy at the end of each day.

Depressive symptoms. Similarly to Study 1 and 2, we asked participants to complete the CES-D to assess participants' current depressive symptoms. High reliability was obtained $(\alpha=.91)$ and the questionnaire showed a moderate correlation with the PHQ-9 completed at prescreening, $r(119)=.47, p<.001$. Ninety-seven participants reported depressive symptom scores above Radloff's (1977) clinical cut-off (i.e. CES-D $\geq 16$ ). Descriptive statistics for the CES-D are presented in Table 6.

Daily diary protocol. To assess the relationship between depression's two main symptoms, sadness and anhedonia, we used a daily diary protocol. For 30 consecutive days, participants received a daily e-mail at 7 PM local time with a hyperlink to a Qualtrics questionnaire. Compliance was high, with an average completion of $93 \%$ of the daily surveys $(S D=7.37 \%)$. In each daily survey participants were asked to rate 11 items measuring daily depressive symptomatology that directly reflected the DSM-5 diagnostic criteria for Major Depressive Disorder (MDD; American Psychiatric Association, 2013). Of interest here, was daily sadness measured as: “Today to what extent did you feel sad?"; and daily anhedonia measured as "Today, to what extent did you experience a diminished interest or pleasure in activities?’. Participants summarized how much they had experienced these two main symptoms of depression on that particular day on a scale ranging from 1 (not at all) to 7 (very much). As both constructs were based on single item measures, assessing reliability was not 
possible. Descriptive statistics for daily sadness, anhedonia and their relationship are presented in Table 6.

Table 6. Means, standard deviations and between-person correlations among all measures in Study 3.

\begin{tabular}{|c|c|c|c|c|c|c|}
\hline & \multirow[b]{2}{*}{ Mean $(S D)$} & \multicolumn{5}{|c|}{ Correlations } \\
\hline & & 1 & 2 & 3 & 4 & 5 \\
\hline 1. CES-D & $29.62(11.60)$ & $.48 * * *$ & & & & \\
\hline 2. Mean daily Sadness & $3.37(1.28)$ & $.48 * * *$ & & & & \\
\hline 3. Mean daily Anhedonia & $3.49(1.21)$ & $.45 * * *$ & $.78 * * *$ & & & \\
\hline 4. SD daily Sadness & $1.24(0.44)$ & .10 & -.07 & -.15 & & \\
\hline 5. SD daily Anhedonia & $1.30(0.47)$ & .10 & -.09 & -.02 & $.73 * * *$ & \\
\hline 6. Sadness-Anhedonia correlation & $.49(0.26)$ & $.32 * * *$ & .14 & .16 & $.41 * * *$ & $.49 * * *$ \\
\hline
\end{tabular}

Note. CES-D scores were obtained at baseline; mean and SD measures of daily Sadness and Anhedonia refer respectively to an individual's average experience of and variability in these symptoms during the daily diary protocol. $* p<.050 . * * p<.010 . * * * p<.001$.

\section{Results and Discussion}

Relation between affective bipolarity and depressive symptoms. Considerable individual differences were observed in the relationship people display between their daily levels of sadness and anhedonia (see Figure 6; range $=-.25-.92)^{27}$. On average, sadness and anhedonia were moderately correlated. For some participants however, sadness and anhedonia were unrelated (i.e. correlation close to .0), while for others they correlated strongly (i.e. correlation close to 1.0$)$.

\footnotetext{
27 Note that a symptom correlation could not be calculated for two individuals due to an absence in variation for
} either reported sadness or anhedonia. 


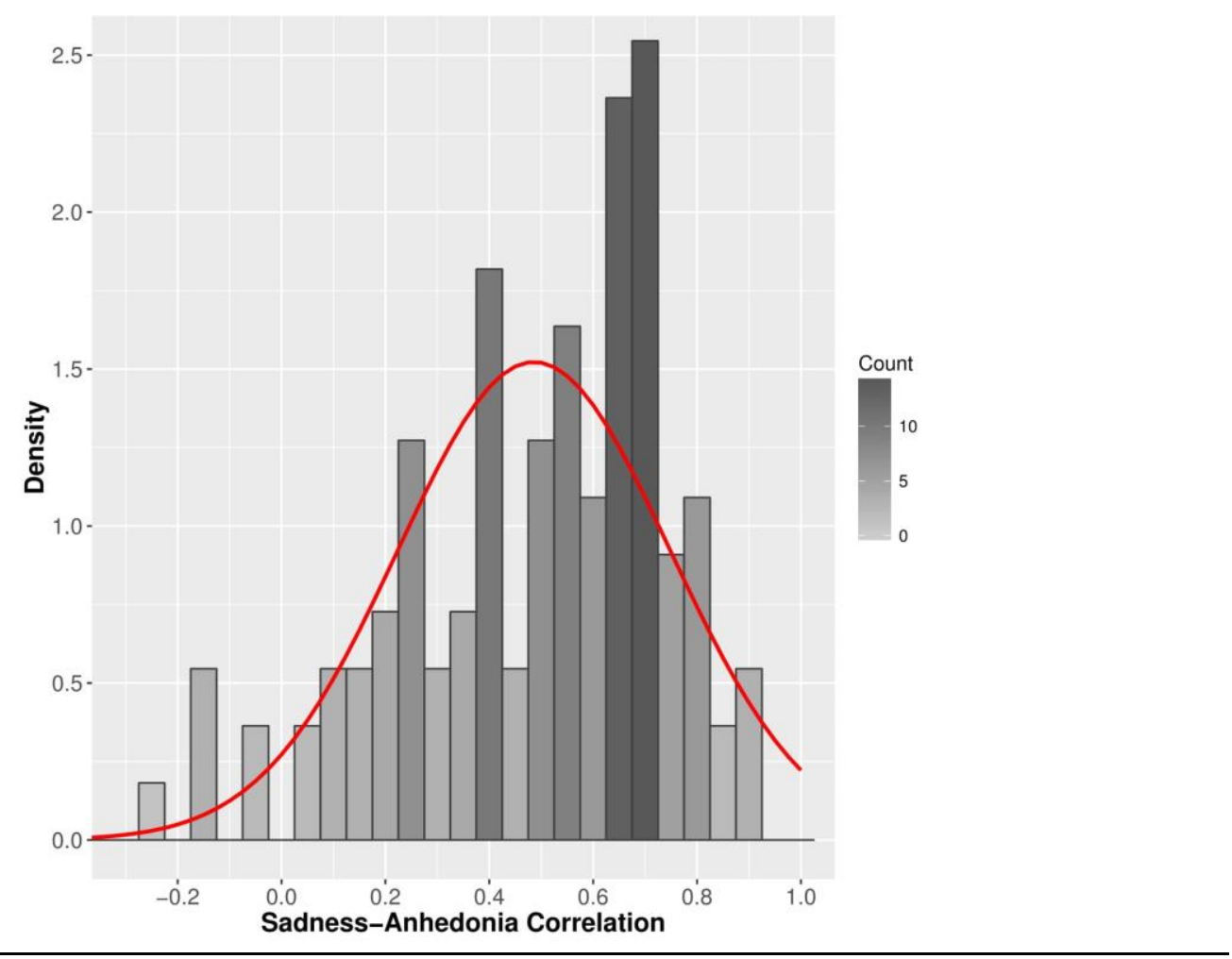

Fig. 6. Density and frequency distribution of participants' Sadness-Anhedonia correlation $(n=110)$. High levels of anhedonia reflect low positive affect, hence the positive correlation coefficients displayed in this figure. The histogram is accompanied by its empirically inferred normal distribution.

The strength of this association showed a meaningful relationship with participants' depressive symptom scores (see Table 6): as participants reported higher CES-D scores, a stronger relationship between daily levels of sadness and anhedonia was observed. This finding extends the range of our conclusions in Study 1 and 2 in two ways. First, depressive symptomatology is characterized by affective bipolarity, irrespective of the time frame in which these affective states are assessed (i.e. daily summary versus momentary assessment; Diener \& Emmons, 1985; Russell \& Carroll, 1999; Watson, 1988). Second, our results replicate with conceptually related manifestations of PA and NA (i.e. depression's core symptoms versus averaging same-valenced discrete emotions).

\section{Controlling for average levels and variability in daily levels of sadness and}

anhedonia. To rule out the possibility again that this correlation was merely driven by participants' mean levels of experienced sadness and anhedonia, we used hierarchical 
multiple regression. In a first model, we let participants' mean levels of sadness and anhedonia predict their CES-D scores (Model 1), followed by a second model, in which the Sadness-Anhedonia correlation was added as an additional predictor (Model 2). Results of these analyses are presented in Table 7. In Model 1 only mean levels of sadness predicted higher depressive symptom severity, with mean levels of anhedonia not contributing to this prediction. Adding the Sadness-Anhedonia correlation in Model 2, led to a significant increase in explained variance, illustrating this symptom relationship shows distinct explanatory power in the prediction of participants' depressive symptoms.

In a final step, next to average symptom levels, we additionally controlled for observed variability in daily sadness and anhedonia (Model 3). This led to similar conclusions, with stronger symptom correlations predicting higher depressive symptom severity $(\beta=.25, p=.012)$. 
Table 7. Hierarchical multiple regression on CES-D scores, exploring the unique main effect of the Sadness-Anhedonia correlation in Study 3.

\begin{tabular}{|c|c|c|c|c|c|c|}
\hline & $R^{2}(\operatorname{adj})$ & $\Delta R^{2}$ & $\Delta F$ & $\beta$ & $t$ & $p$ \\
\hline Model 1 & .22 & .23 & $16.00 * * *$ & & & \\
\hline Intercept & & & & & 10.35 & $<.001$ \\
\hline Mean daily Sadness & & & & .33 & 2.53 & .013 \\
\hline Mean daily Anhedonia & & & & .18 & 1.38 & .170 \\
\hline Model 2 & .28 & .07 & $9.71 * *$ & & & \\
\hline Intercept & & & & & 8.68 & $<.001$ \\
\hline Mean daily Sadness & & & & .32 & 2.55 & .012 \\
\hline Mean daily Anhedonia & & & & .15 & 1.17 & .244 \\
\hline Sadness-Anhedonia correlation & & & & .26 & 3.12 & .002 \\
\hline Model 3 & .27 & .003 & .26 & & & \\
\hline Intercept & & & & & 6.15 & $<.001$ \\
\hline Mean daily Sadness & & & & .29 & 2.21 & .030 \\
\hline Mean daily Anhedonia & & & & .18 & 1.32 & .190 \\
\hline SD daily Sadness & & & & .09 & 0.71 & .480 \\
\hline SD daily Anhedonia & & & & -.06 & -0.41 & .680 \\
\hline Sadness-Anhedonia correlation & & & & .25 & 2.54 & .012 \\
\hline
\end{tabular}
Note. $* p<.050 . * * p<.010 . * * * p<.001$.

In conclusion, Study 3 extended the concurrent conclusions of Study 1 and 2 by replicating our findings in a sample with elevated depressive symptom scores, using different, but conceptually related indicators of positive and negative affect that were assessed on a different timescale. Daily levels of sadness and anhedonia were more strongly related in participants with higher depressive symptom scores, suggesting a more bipolar experience of 
positive and negative affect for these individuals. Again, this relation remained apparent after controlling for average levels of and variability in these symptoms.

\section{General Discussion}

The present research set out to explore the bipolarity of affect in relation to experiencing depressive symptoms. Across three daily life studies we found converging evidence that individual differences in how positive and negative affect are structured in people's experience are meaningfully related to levels of depressive symptom severity. Specifically, we consistently demonstrated that experiencing more depressive symptoms is associated with a stronger bipolar experience of positive and negative affect. A meta-analysis of all studies showed that participants' displayed affective bipolarity was correlated .234 with their depressive symptoms, Fisher's $Z r=.238, \mathrm{SE}=0.036,95 \% \mathrm{CI}[.167, .309]$. This relation holds even after controlling for mean levels of experienced PA and NA, Fisher's $Z r=.165$, $\mathrm{SE}=0.036,95 \% \mathrm{CI}[.094, .236]$, and to some degree also after taking variability in these affective states into account, Fisher's $Z r=.153, \mathrm{SE}=0.036,95 \% \mathrm{CI}[.083, .227]$. Furthermore, this association seems robust across different important factors that are known to influence the relation between positive and negative affect and shows specificity towards depressive symptomatology, in comparison with anxiety symptoms. In the next paragraphs, we discuss the meaning of these findings and formulate noteworthy limitations of the present studies that future research should address.

\section{Depressive symptoms, more than Altered Mean Levels of Positive and Negative Affect}

To our knowledge, this is the first study to investigate the within-person relation between these two affective states in the experience of depressive symptoms. Traditionally, depressive symptomatology is generally understood to be marked by altered average levels of positive and negative affect (Clark \& Watson, 1991; Watson \& Clark, 1984; Watson et al., 1988), inherently reflected by the two conceptually related core symptoms of depression, 
depressed mood and anhedonia. Furthermore, depressive symptoms have also been linked to increased variability in positive and negative emotional responding (Houben et al., 2015; Koval, Pe, et al., 2013). The current findings, however, indicate that depressive symptomatology is additionally characterized by changes in the way these affective states are structured, revealing a new manifestation of how emotional experiences are altered in relation to these symptoms.

First, our findings indicate reduced emotional complexity in people with depressive complaints. When experiencing depressive symptoms, one's emotional life tends to be more unidimensional, with increases in NA being functionally equivalent to decreases in PA, and vice versa. This observed emotional simplification is in line with predictions made by both the control-process model (Carver \& Scheier, 1990) and the DMA (Zautra et al., 1997). In this first model, emotional simplification may be a consequence of depressed people's lack of goal specificity (e.g. Carver \& Ganellen, 1983). Depression is not only associated with pursuing fewer goals, they are also more abstract and ill-defined (e.g. Beck, 2002; Dickson et al., 2016). These broad and unspecified objectives may leave one's emotional life to be less complex and diversified, as an affective experience associated with a single goal attainment is usually strictly bipolar (Carver, 2006; Carver \& Scheier, 1990). In contrast, non-depressed individuals generally pursue multiple goals in life, making it possible to experience positive and negative affect more independently. Following the DMA, emotion simplification may also be the result of the constraints depressive symptoms place on an individual's cognitive resources (e.g. DeBattista, 2005; Zautra et al., 2002). Depressive symptoms are burdensome (Fossati et al., 2002; Gotlib \& Joormann, 2010; Kircanski et al., 2012) and are likely to inhibit normal functioning, which makes deliberate and thorough information processing difficult. Hence, when confronted with emotional stimuli, individuals who experience depressive symptoms may respond in a basic and heuristic manner, only being capable to pay attention to 
one valence (i.e. often the negative one; see Baert, De Raedt, \& Koster, 2010). One might argue, however, that although depressive symptoms are associated with greater affective bipolarity, they may involve richer and more complex experiences of negative emotions. Yet, research on negative emotion differentiation in depression suggests the opposite, showing that depressed individuals tend not to differentiate between different negative emotions (Demiralp et al., 2012; Erbas, Ceulemans, Pe, Koval, \& Kuppens, 2014). In addition to less fine-grained distinctions between equally valenced emotions (i.e. poor emotional granularity; Lindquist \& Barrett, 2008), the present findings illustrate that people with depressive symptoms also process the relationship between oppositely valenced affective states in a more bipolar, heuristic way.

Second, affective bipolarity also implies emotional inflexibility. Individuals who experience depressive symptoms lack the capacity to adaptively regulate their emotions to situational demands (Houben et al., 2015; Kuppens et al., 2012; van de Leemput et al., 2014). This deficit may be aggravated by the inability to experience PA and NA relatively independently, as it hampers subtle emotional responding towards ambiguous or mixed events. When positive and negative affective states are bipolar opposites, one's emotional repertoire becomes narrowed, perhaps preventing a depressed person from experiencing the most appropriate emotion in a certain situation (e.g. feeling bittersweet during a farewell).

While future research should definitely pinpoint the exact mechanisms that are involved in pushing a person's affective system towards bipolarity, results of Study 2 indicate depressive symptoms precede rather than follow from affective bipolarity. Although causality cannot be inferred from our longitudinal design, current findings are consistent with a DMAstudy in which experimentally induced stress led to an increase in affective bipolarity (Zautra et al., 1997). In this sense, one could argue that the burden depressive symptoms place on an individual is likely to create a bipolar affective system (e.g. Gotlib \& Joorman, 2010). 


\section{Beyond Notable Factors that Influence the Relation between Positive and Negative Affect}

Theoretically, the current findings add to our knowledge about which factors (e.g. construct operationalization, assessed time frame, etc.) have an effect on the within-person structure of emotion. Our results may further encourage emotion researchers to also take into account individual differences in the affective relation, and to unravel potentially meaningful mechanisms and covariates explaining these differences.

Importantly, assessing how individual differences in depressive symptomatology interact with two other significant factors that are known to impact the PA-NA relation, showed the link between affective bipolarity and depressive symptoms was quite robust across these other determinants. First, depressive symptomatology was characterized by affective bipolarity, irrespective of how our affective constructs were operationalized (Barrett \& Russell, 1998; Russell \& Carroll, 1999). In Study 1 and 2, discrete emotion words were averaged to create a momentary PA and NA construct. Multiverse analyses, in which multiple PA-NA relations were computed based on different item sets, indicated that affective bipolarity was related to depressive symptoms largely irrespective of the items that were used to indicate positive and negative feelings. This was also replicated when using sadness and anhedonia as conceptually related markers for high negative and low positive affect, respectively, in Study 3. Together, this indicates the robustness of our findings across different items and operationalizations of PA and NA. It implies, for instance, that a possible confound of arousal is minimal, as different PA-NA relations also capture different arousal associations (e.g. relaxed-depressed versus relaxed-angry). These results suggest also that the degree to which emotion words truly represent semantic antonyms did not drastically impact our conclusions (e.g. happy-sad versus happy-anxious).

Second, the time frame in which participants' positive and negative affect was assessed did not influence the relation with depressive symptoms either (Diener \& Emmons, 
1985; Russell \& Carroll, 1999; Watson, 1988). While Study 1 and 2 captured momentary experiences of PA and NA (i.e. how do you feel at the moment?), the time scale in Study 3 was longer, with the assessment of a daily summary of these affective states instead (i.e. how did you feel today?).

Although the consistency of our findings strengthens the confidence in our conclusions, future research should replicate our findings with different sets of item terms, even longer time frames, and investigate the possible impact of other factors influencing the relation between PA and NA, such as response type format (Warr et al., 1983), the inclusion of measurement error (Green et al., 1993), and level of analysis (within- versus between-person level; Brose, Voelkle, et al., 2015), to explore the boundary conditions of the present results.

Finally, in a similar vein, the relation between depressive symptoms and affective bipolarity was evident across the various depressive symptom constellations participants displayed. A depressive symptom score can be composed of different symptoms, with in extremis two individuals reporting a similar elevated score, but not sharing a single symptom (e.g. Fried, 2015; Fried \& Nesse, 2015). The fact that depressive symptomatology was characterized by affective bipolarity irrespective of its symptom constellation, suggests it may serve as a general marker of these symptoms, and is not particularly tied to specific symptom compositions $^{28}$. Yet, future research could look into particular symptoms or subtypes of depression for which affective bipolarity is notably strong. Based on the DMA (Zautra et al., 1997), for example, one could hypothesize that a bipolar experience of PA and NA is especially apparent in depressed individuals who tend to ruminate frequently, as repetitive negative thinking is likely to burden an individual's cognitive recourses (Christopher \& Macdonald, 2005).

\section{Depressive Symptoms, Clinical Depression or General Emotional Disturbances?}

\footnotetext{
${ }^{28}$ This idea is strengthened by the fact that this relation was evident across various operationalizations of our depressive construct (i.e. CES-D, DASS-21 depression subscale, or a combination of both).
} 
Is affective bipolarity specific to depressive symptoms or is it linked to various types of complaints, reflecting general emotional maladjustment instead? Across studies, affective bipolarity was related to general symptoms of distress. This is in line with our predictions based on the DMA (Zautra et al., 1997), stating distress or high levels of negative affect may push one's affective structure towards bipolarity. However, when additionally taking into account symptoms of depression or anxiety, affective bipolarity was only apparent in light of depressive, but not anxiety complaints. Although both symptom types are equally distressing and are both characterized by high levels of negative affect, this finding is in line with our predictions based on the tripartite model (Clark \& Watson, 1991). Following this model, positive affect is only impaired in depressive, not anxiety symptoms. Hence, as both affective states are only altered in the opposite direction (i.e. high NA, low PA) in the light of depressive symptoms, this may explain why affective bipolarity was only related to (the distress associated) with depressive symptomatology. In contrast, unaffected PA levels, as generally the case when suffering from anxiety symptoms, suggest both affective states can still operate separately from each other, indicating affective independence (Barlow et al., 1996; Watson \& Kendall, 1989). Combined, these results suggest affective bipolarity is not a marker of general emotional disturbances, but rather shows a certain specificity towards depressive symptoms, in comparison with anxiety complaints.

Finally, we believe that conclusions with respect to clinical depression or MDD should be tempered, as extrapolating our results into the clinical realm may be unwarranted. Highend clinically depressed patients differ in important aspects from sub-clinical individuals displaying normal variation in these symptoms (e.g. in terms of global functioning, needing treatment, etc.; Coyne, 1994). Indeed, as we only used data from non-clinical samples and all participants in the reported studies were still relatively healthy (even in Study 3), this cautions us to make claims about clinical depression. Future research should replicate the current 
findings in a clinical sample, with clinicians assessing current and previous episodes of MDD (as opposed to solely relying on self-report measures in our studies).

\section{Conclusion}

Experiencing depressive symptoms involves more than altered mean levels of positive and negative affect. Next to an increase in negativity and a decrease in positivity, we found that the way these affective states are structured within individuals is also modified when feeling depressed in terms of a more bipolar experience of positive and negative affect, reflecting reduced emotional complexity and flexibility. 
THE BIPOLARITY OF AFFECT AND DEPRESSIVE SYMPTOMS

\section{Declaration of Conflicting Interests}

The authors declare no conflict of interest with respect to the authorship or the publication of this article. 


\section{References}

American Psychiatric Association. (2013). Diagnostic and statistical manual of mental disorders (5th ed.). Arlington, VA: American Psyciatric Association.

Arditte, K. A., Çek, D., Shaw, A. M., \& Timpano, K. R. (2015). The importance of assessing clinical phenomena in Mechanical Turk research. Psychological Assessment, 28, 684691. http://doi.org/10.1037/pas0000217

Baert, S., De Raedt, R., \& Koster, E. H. W. (2010). Depression-related attentional bias: The influence of symptom severity and symptom specificity. Cognition and Emotion, 24, 1044-1052. http://doi.org/10.1080/02699930903043461

Bagozzi, R. P., Wong, N., \& Yi, Y. (1999). The role of culture and gender in the relationship between positive and negative affect. Cognition and Emotion, 13, 641-672. http://doi.org/10.1080/026999399379023

Barlow, D. H., Chorpita, B. F., \& Turovsky, J. (1996). Perspectives on anxiety, panic, and fear. In D. J. Hansen (Ed.), Nebraska Symposium on Motivation (pp. 251-328). Lincoln, NE: University of Nebraska Press.

Barrett, L. F., \& Russell, J. A. (1998). Independence and bipolarity in the structure of current affect. Journal of Personality and Social Psychology, 74, 967-984. http://dx.doi.org/10.1037/0022-3514.74.4.967

Beck, A. T. (2002). Cognitive models of depression. In R. L. Leahy \& E. Dowd, Thomas (Eds.), Clincal Advances in Cognitive Psychotherapy: Theory and Application (pp. 2961). New York, NY: Springer Publshing Company.

Bradburn, N. M. (1969). The Structure of Psychological Well-Being. Chicago, IL: Aldine. Brose, A., de Roover, K., Ceulemans, E., \& Kuppens, P. (2015). Older adults' affective experiences across 100 days are less variable and less complex than younger adults'. Psychology and Aging, 30, 194-208. http://dx.doi.org/10.1037/a0038690 
Brose, A., Voelkle, M. C., Lövdén, M., Lindenberger, U., \& Schmiedek, F. (2015). Differences in the between-person and within-person structures of affect are a matter of degree. European Journal of Personality, 29, 55-71. http://dx.doi.org/10.1002/per.1961

Brose, A., Wichers, M., \& Kuppens, P. (2017). Daily stressful experiences precede but do not succeed depressive symptoms: Results from a longitudinal experience sampling study. Journal of Social and Clinical Psychology, 36, 196-220. https://doi.org/10.1521/jscp.2017.36.3.196.

Brown, T. A., Chorpita, B. F., Korotitsch, W., \& Barlow, D. H. (1997). Psychometric properties of the depression anxiety stress scales (DASS) in clinical samples. Behavioral Research and Therapy, 35, 79-89. http://dx.doi.org/10.1348/014466506X158996

Cacioppo, J. T., Gardner, W. L., \& Berntson, G. G. (1997). Beyond bipolar conceptualizations and measures: The case of attitudes and evaluative space. Personality and Social Psychology Review, 1, 3-25. https://doi.org/10.1207/s15327957pspr0101_2

Carstensen, L. L., Turan, B., Scheibe, S., Ram, N., Ersner-hershfield, H., Brooks, K. P., \& Nesselroade, J. R. (2011). Emotional experience improves with age: Evidence based on over 10 years of experience sampling. Psychology and Aging, 26, 21-33. http://doi.org/10.1037/a0021285.Emotional

Carver, C. S. (2004). Negative affects deriving from the behavioral approach system. Emotion, 4, 3-22. http://doi.org/10.1037/1528-3542.4.1.3

Carver, C. S. (2006). Approach, avoidance, and the self-regulation of affect and action. Motivation and Emotion, 30, 105-110. http://doi.org/10.1007/s11031-006-9044-7

Carver, C. S., \& Ganellen, R. J. (1983). Depression and components of self-punitiveness: High standards, self-criticism, and overgeneralization. Journal of Abnormal Psychology, 92, 330-337. http://dx.doi.org/10.1037/0021-843X.92.3.330

Carver, C. S., \& Scheier, M. F. (1990). Origins and functions of positive and negative affect: 
A control-process view. Psychological Review, 97, 19-35.

http://dx.doi.org/10.1037/0033-295X.97.1.19

Casler, K., Bickel, L., \& Hackett, E. (2013). Computers in human behavior separate but equal? A comparison of participants and data gathered via amazon's MTurk, social media, and face-to-face behavioral testing. Computers in Human Behavior, 29, 2156 2160. http://doi.org/10.1016/j.chb.2013.05.009

Christopher, G., \& Macdonald, J. (2005). The impact of clinical depression on working memory. Cognitive Neuropsychiatry, 10, 379-399. http://doi.org/10.1080/13546800444000128

Clark, L. A., \& Watson, D. (1991). Tripartite model of anxiety and depression: Psychometric evidence and taxonomic implications. Journal of Abnormal Psychology, 100, 316-336. http://dx.doi.org/10.1037/0021-843X.100.3.316

Coifman, K. G., Bonanno, G. A., \& Rafaeli, E. (2007). Affect dynamics, bereavement and resilience to loss. Journal of Happiness Studies. 8, 371-391. http://dx.doi.org/10.1007/s10902-006-9014-5

Coyne, J. C. (1994). Self-reported distress: Analog or ersatz depression? Psychological Bulletin, 116, 29-45. http://dx.doi.org/10.1037/0033-2909.116.1.29

Csikszentmihalyi, M., \& Larson, R. (1987). Validity and reliability of the experiencesampling method. Journal of Nervous and Mental Disorders, 175, 526-536. http://dx.doi.org/10.1097/00005053-198709000-00004

Davis, M. C., Zautra, A. J., \& Smith, B. (2004). Chronic pain, stress, and the dynamics of affective differentiation. Journal of Personality, 72, 1133-1159. http://doi.org/10.1111/j.1467-6494.2004.00293.x

DeBattista, C. (2005). Executive dysfunction in major depressive disorder. Expert Review in Neurotherapeutics, 5, 79-84. https://doi.org/10.1586/14737175.5.1.79 
Dejonckheere, E., Bastian, B., Fried, E. I., Murphy, S., \& Kuppens, P. (2017). Perceiving social pressure not to feel negative predicts depressive symptoms in daily life. Depression and Anxiety, 34, 836-844. http://doi.org/10.1002/da.22653

Demiralp, E., Thompson, R. J., Mata, J., Jaeggi, S. M., Buschkuehl, M., Barrett, L. F., ... Jonides, J. (2012). Feeling blue or turquoise? Emotional differentiation in Major Depressive Disorder. Psychological Science, 23, 1410-1416. http://doi.org/10.1177/0956797612444903

Dickson, J. M., Moberly, N. J., O’Dea, C., \& Field, M. (2016). Goal fluency, pessimism and disengagement in depression. PloS One, 11, e0166259. http://doi.org/10.1371/journal.pone.0166259

Diener, E. (1999). Introduction to the special section on the structure of emotion. Journal of Personality and Social Psychology, 76, 803-804. http://psycnet.apa.org/doi/10.1037/0022-3514.76.5.803

Diener, E., \& Iran-Nejad, A. (1986). The relationship in experience between various types of affect. Journal of Personality and Social Psychology, 50, 1031-1038. http://psycnet.apa.org/doi/10.1037/0022-3514.50.5.1031

Diener, E., \& Emmons, R. A. (1985). The independence of positive and negative affect. Journal of Personality and Social Psychology, 47, 1105-1117. http://psycnet.apa.org/doi/10.1037/0022-3514.47.5.1105

Erbas, Y., Ceulemans, E., Koval, P., \& Kuppens, P. (2015). The role of valence focus and appraisal overlap in emotion differentiation. Emotion, 15, 373-382. http://doi.org/http://dx.doi.org/10.1037/emo0000039

Erbas, Y., Ceulemans, E., Pe, M. L., Koval, P., \& Kuppens, P. (2014). Negative emotion differentiation: Its personality and well-being correlates and a comparison of different assessment methods. Cognition and Emotion, 28, 1196-1213. 
BIPOLARITY OF AFFECT AND DEPRESSIVE SYMPTOMS

http://doi.org/10.1080/02699931.2013.875890

Feldman, L. A. (1995). Valence focus and arousal focus: Individual differences in the structure of affective experience. Journal of Personality and Social Psychology, 69, 153166. http://psycnet.apa.org/doi/10.1037/0022-3514.69.1.153

Ferrari, A. J., Charlson, F. J., Norman, R. E., Patten, S. B., Freedman, G., Murray, C. J. L., ... Whiteford, H. A. (2013). Burden of depressive disorders by country, sex, age, and year: Findings from the global burden of disease study 2010. PloS Medicine, 10, 1-11. http://doi.org/10.1371/journal.pmed.1001547

Fisher, C. D., \& To, M. L. (2012). Using experience sampling methodology in organizational behavior. Journal of Organizational Behavior, 38, 1-13. http://doi.org/10.1002/job.1803

Fossati, P., Ergis, A. M., Allilaire, J. F. (2002). Executive functioning in unipolar depression: A review. Encephale, 28, 97-107.

Fried, E. I. (2015). Problematic assumptions have slowed down depression research: why symptoms, not syndromes are the way forward. Frontiers in Psychology, 6, 1-11. http://doi.org/10.3389/fpsyg.2015.00309

Fried, E. I., \& Nesse, R. M. (2015). Depression sum-scores don't add up: Why analyzing specific depression symptoms is essential. BioMed Central Medicine, 13, 1-11. http://doi.org/10.1186/s12916-015-0325-4

Gotlib, I. H., \& Joormann, J. (2010). Cognition and depression: Current status and future directions. Annual Review of Clinical Psychology, 27, 285-312. http://doi.org/10.1146/annurev.clinpsy.121208.131305

Green, D. P., Goldman, S. L., \& Salovey, P. (1993). Measurement error masks bipolarity in affect ratings. Journal of Personality and Social Psychology, 64, 1029-1041.

Grossmann, I., Huynh, A. C., \& Ellsworth, P. C. (2015). Emotional complexity: Clarifying definitions and cultural correlates. Journal of Personality and Social Psychology, 2, 1- 
22. http://dx.doi.org/10.1037/pspp0000084

Grühn, D., Lumley, M. A., \& Labouvie-vief, G. (2013). Time-based indicators of emotional complexity: Interrelations and correlates. Emotion, 13, 226-237. http://doi.org/10.1037/a0030363.Time-Based

Hamilton, J. D. (1994). Time Series Analysis. Princeton, NJ: Princeton University Press.

Houben, M., Van Den Noortgate, W., \& Kuppens, P. (2015). The relation between short-term emotion dynamics and psychological well-being: A meta-analysis. Psychological Bulletin, 141, 901-930. https://doi.org/10.1037/a0038822

Ingram, R. E., \& Siegle, G. J. (2009). Methodological issues in the study of depression. In I. H. Gotlib \& H. C.L. (Eds.), Handbook of Depression (pp. 69-92). New York, NY: The Guilford Press.

Kircanski, K., Joormann, J., \& Gotlib, I. H. (2012). Cognitive aspects of depression. Wiley Interdisciplinary Review of Cognitive Sciences, 3, 301-313. http://doi.org/10.1002/wcs. 1177

Klein, D. (1984). Depression and anhedonia. In D. Clark \& J. Fawcett (Eds.), Anhedonia and affect deficit states (pp. 1-34). New York, NY: PMA Publishing.

Koval, P., Brose, A., Pe, M. L., Houben, M., Erbas, Y., Champagne, D., \& Kuppens, P. (2015). Emotional inertia and external events: The roles of exposure, reactivity, and recovery. Emotion, 15, 625-363. http://dx.doi.org/10.1037/emo0000059.

Koval, P., Ogrinz, B., Kuppens, P., Van den Bergh, O., Tuerlinckx, F., \& Sütterlin, S. (2013). Affective instability in daily life is predicted by resting heart rate variability. PLoS One, 8, e81536. http://doi.org/10.1371/journal.pone.0081536.

Koval, P., Pe, M. L., Meers, K., \& Kuppens, P. (2013). Affect dynamics in relation to depressive symptoms: Variable, unstable or inert? Emotion, 13, 1132-1141. http://doi.org/10.1037/a0033579 
Kroenke, K., Spitzer, R. L., \& Williams, J. B. (2001). The PHQ-9: Validity of a brief depression severity measure. Journal of General Internal Medicine, 16, 606-613. http://doi.org/10.1046/j1525-14972001016009606x

Kuppens, P., Sheeber, L. B., Yap, M. B. H., Whittle, S., Simmons, J. G., \& Allen, N. B. (2012). Emotional inertia prospectively predicts the onset of depressive disorder in adolescence. Emotion, 12, 283-289. http://doi.org/10.1037/a0025046

Larsen, J. T., Hershfield, H. E., Stastny, B. J., \& Hester, N. (2017). On the relationship between positive and negative affect: Their correlation and their co-occurrence. Emotion, 17, 1-14. https://doi.org/10.1037/emo0000231

Larsen, J. T., McGraw, A. P., \& Cacioppo, J. T. (2001). Can people feel happy and sad at the same time? Journal of Personality and Social Psychology, 81, 684-696. http://doi.org/10.1037//0022-3514.81.4.684

Lindquist, K. A., \& Barrett, L. F. (2008). Emotional complexity. In M. Lewis, J. M. HavilandJones, \& L. F. Barrett (Eds.), The handbook of Emotions (pp. 1-31). New York, NY: The Guilford press.

Lovibond, S. H., \& Lovibond, P. F. (1995). Manual for the depression anxiety stress scales. Sydney, Australia: The Psychology Foundation of Australia.

Moors, A., Houwer, J. De, Hermans, D., Winne, J. De, \& Brysbaert, M. (2013). Norms of valence, arousal, dominance, and age of acquisition for 4.300 Dutch words. Behavioural Research, 45, 169-177. http://doi.org/10.3758/s13428-012-0243-8

Nezlek, J. B. (2016). A practical guide to understanding reliability in studies of within-person variability. Journal of Research in Personality. http://doi.org/10.1016/j.jrp.2016.06.020

Nowlis, V., \& Nowlis, H. H. (1956). The description and analysis of mood. In Annals of the New York Academy of Sciences (pp. 345-355). New York, NY: New York Academy of Sciences. 
Ong, A. D., Zautra, A. J., \& Finan, P. H. (2017). Inter- and intra-individual variation in emotional complexity: Methodological considerations and theoretical implications. Current Opinion in Behavioral Sciences, 15, 22-26.

http://doi.org/10.1016/j.cobeha.2017.05.018

Pe, M. L., Brose, A., Gotlib, I. H., \& Kuppens, P. (2016). Affective updating ability and stressful events interact to prospectively predict increases in depressive symptoms over time. Emotion, 16, 73-82. https://doi.org/10.1037/emo0000097

Pe, M. L., Kircanski, K., Thompson, R. J., Bringmann, L. F., Tuerlinckx, F., Mestdagh, M., ... Gotlib, I. H. (2015). Emotion-network density in major depressive disorder. Psychological Science, 3, 292-300. http://doi.org/10.1177/2167702614540645

Pe, M. L., Koval, P., Houben, M., Erbas, Y., Champagne, D., \& Kuppens, P. (2015). Updating in working memory predicts greater emotion reactivity to and facilitated recovery from negative emotion-eliciting stimuli. Frontiers in Psychology, 6, 1-13. http://doi.org/10.3389/fpsyg.2015.00372.

Pe, M. L., Koval, P., \& Kuppens, P. (2013). Executive well-being: Updating of positive stimuli in working memory is associated with subjective well-being. Cognition, 126, 335-340. http://dx.doi.org/10.1016/j.cognition.2012.10.002.

Pitzer, L. M., \& Bergeman, C. S. (2014). Synchrony in affect among stressed adults: The Notre Dame widowhood study. Journals of Gerontology, Series B: Psychological Sciences and Social Sciences, 69, 29-39. http://doi.org/10.1093/geronb/gbt026

Potter, P. T., Zautra, A. J., \& Reich, J. W. (2000). Stressful events and information processing dispositions moderate the relationship between positive and negative affect: Implications for pain patients. Annals of Behavioral Medicine, 22, 191-198. https://doi.org/10.1007/BF02895113

Radloff, L. S. (1977). The CES-D scale: A self-report depression scale for research in the 
BIPOLARITY OF AFFECT AND DEPRESSIVE SYMPTOMS

general population. Applied Psychological Measurement, 1, 384-401.

http://doi.org/doi:10.1177/014662167700100306

Rafaeli, E., Rogers, G. M., \& Revelle, W. (2007). Affective synchrony: Individual differences in mixed emotions. Personality and Social Psychology Bulletin, 33, 915-932. http://doi.org/10.1177/0146167207301009

Ready, R. E., Åkerstedt, A. M., \& Mroczek, D. K. (2011). Emotional complexity and emotional well-being in older adults: Risks of high neuroticism. Aging \& Mental Health, 16, 17-26. http://doi.org/10.1080/13607863.2011.602961

Reich, J. W., Zautra, A. J., \& Davis, M. (2003). Dimensions of affect relationships: Models and their integrative implications. Review of General Psychology, 7, 66-83. http://doi.org/10.1037/1089-2680.7.1.66

Russell, J. A. (1979). Affective space is bipolar. Journal of Personality and Social Psychology, 37, 345-356. http://doi.org/10.1037/0022-3514.37.3.345

Russell, J. A. (1980). A circumplex model of affect. Journal of Personality and Social Psychology, 39, 1161-1178. http://doi.org/10.1037/h0077714

Russell, J. A., \& Carroll, J. M. (1999). On the bipolarity of positive and negative affect. Psychological Bulletin, 125, 3-30. http://doi.org/10.1037//0033-2909.125.1.3

Rydmark, I., Wahlberg, K., Ghatan, P. H., Modell, S., Nygren, Å., Ingvar, M., ... Heilig, M. (2006). Neuroendocrine, cognitive and structural imaging characteristics of women on longterm sickleave with job stress-induced depression. Biological Psychiatry, 60, 867873. http://doi.org/10.1016/j.biopsych.2006.04.029

Schimmack, U. (2001). Pleasure, displeasure, and mixed feelings: Are semantic opposites mutually exclusive? Cognition and Emotion, 15, 81-97. http://doi.org/10.1080/02699930126097

Schmukle, S. C., Egloff, B., \& Burns, L. R. (2002). The relationship between positive and 
negative affect in the positive and negative affect schedule. Journal of Research in Psychology, 36, 463-475. https://doi.org/10.1016/S0092-6566(02)00007-7

Schuurman, N. K., Ferrer, E., de Boer-Sonnenschein, \& M., Hamaker, E. L. (2016). How to compare cross-lagged association in a multilevel autoregressive model. Psychological Methods, 21, 206-221.

Shim, R. S., Baltrus, P., Ye, J., \& Rust, G. (2011). Prevalence, treatment, and control of depressive symptoms in the united states: Results from the national health and nutrition examination survey (NHANES), 2005-2008. Journal of the American Board of Family Medicine, 24, 33-38. http://doi.org/10.3122/jabfm.2011.01.100121.Prevalence

Steegen, S., Tuerlinckx, F., Gelman, A., \& Vanpaemel, W. (2016). Increasing transparency through a multiverse analysis. Perspectives on Psychological Science, 11, 702-712. http://doi.org/10.1177/1745691616658637

Tellegen, A. (1985). Structures of mood and personality and their relevance to assessing anxiety, with an emphasis on self-report. In A. H. Tuma \& J. D. Maser (Eds.), Anxiety and Anxiety Disorders (pp. 681-706). Hillsdale, NJ: Erlbaum.

Thayer, R. E. (1989). The biopsychology of mood and arousal. New York, NY: Oxford University Press.

Treadway, M. T., \& Zald, D. H. (2013). Parsing Anhedonia: Translational models of rewardprocessing deficits in psychopathology. Current Directions in Psychological Science. 22, 244-249. http://doi.org/10.1177/0963721412474460

van de Leemput, I. A., Wichers, M., Cramer, A. O. J., Borsboom, D., Tuerlinckx, F., Kuppens, P., ... Scheffer, M. (2014). Critical slowing down as early warning for the onset and termination of depression. Proceedings of the National Academy of Sciences, 111, 87-92. http://doi.org/10.1073/pnas.1312114110

Warr, P., Barter, J., \& Brownbridge, G. (1983). On the independence of positive and negative 
affect. Journal of Personality and Social Psychology, 44, 644-651.

http://psycnet.apa.org/doi/10.1037/0022-3514.44.3.644

Watson, D. (1988). The vicissitudes of mood measurement: Effects of varying descriptors, time frames, and response formats on measures of positive and negative affect. Journal of Personality and Social Psychology, 55, 128-141.

http://psycnet.apa.org/doi/10.1037/0022-3514.55.1.128

Watson, D., \& Clark, L. A. (1984). Negative affectivity: The disposition to experience aversive emotional states. Psychological Bulletin, 96, 465-490.

http://doi.org/10.1037/0033-2909.96.3.465

Watson, D., \& Clark, L. A. (1997). Measurement and mismeasurement of mood: Recurrent and emergent issues. Journal of Personality Assessment, 68, 267-296. https://doi.org/10.1207/s15327752jpa6802_4

Watson, D., Clark, L. A., \& Carey, G. (1988). Positive and negative affectivity and their relation to anxiety and depressive disorders. Journal of Abnormal Psychology, 97, 346353. https://doi.org/10.1037/0021-843X.97.3.346

Watson, D., Clark, L. A., \& Tellegen, A. (1988). Development and validation of brief measures of positive and negative affect: The PANAS scales. Journal of Personality and Social Psychology, 54, 1063-1070. https://doi.org/10.1037//0022-3514.54.6.1063

Watson, D., \& Kendall, P. C. (1989). Understanding anxiety and depression: their relation to negative and positive affective states. In P. C. Kendall \& D. Watson (Eds.), Anxiety and depression: Distinctive and overlapping features (pp. 3-26). New York, NY: Academic Press.

Watson, D., \& Tellegen, A. (1985). Toward a consensual structure of mood. Psychological Bulletin, 98, 219-235. http://psycnet.apa.org/doi/10.1037/0033-2909.98.2.219

Watson, D., Wiese, D., Vaidya, J., \& Tellegen, A. (1999). The two general activation systems 
of affect: Structural findings, evolutionairy considerations, and psychobiological evidence. Journal of Personality and Social Psychology, 76, 820-838. http://psycnet.apa.org/doi/10.1037/0022-3514.76.5.820

Williams, J., Peeters, F., \& Zautra, A. J. (2004). Differential affect structure in depressive and anxiety disorders. Anxiety, Stress, \& Coping, 17, 321-330. http://dx.doi.org/10.1080/10615800412331318634

Yik, M. S. M., Russell, J. A., \& Barrett, L. F. (1999). Structure of self-reported current affect: Integration and beyond. Journal of Personality and Social Psychology, 77, 600-617. http://psycnet.apa.org/doi/10.1037/0022-3514.77.3.600

Zautra, A. J., Affleck, G. G., Tennen, H., Reich, J. W., \& Davis, M. C. (2005). Dynamic approaches to emotions and stress in everyday life: Bolger and Zuckerman reloaded with positive as well as negative affects. Journal of Personality, 73, 1511-1538. https://dx.doi.org/10.1111\%2Fj.0022-3506.2005.00357.x

Zautra, A. J., Berkhof, J., \& Nicolson, N. A. (2002). Changes in affect interrelations as a function of stressful events. Cognition and Emotion, 16, 309-318. http://doi.org/10.1080/02699930143000257

Zautra, A. J., Potter, P. T., \& Reich, J. W. (1997). The independence of affects is contextdependent: An integrative model of the relationship between positive and negative affect. In M. P. Lawton \& S. K.W. (Eds.), Annual Review of Gerontology and Geriatrics (pp. 75-102). New York, NY: Springer Publishing Company.

Zautra, A. J., Reich, J. W., Davis, M. C., Potter, P. T., \& Nicolson, N. A. (2000). The role of stressful events in the relationship between positive and negative affects: Evidence from field and experimental studies. Journal of Personality, 68, 927-951. http://psycnet.apa.org/doi/10.1111/1467-6494. 


\section{Supplementary Materials}

Supplementary Tables $1-3$ present all multilevel models with person-mean standardized PA and NA, both variables either being predictor or outcome. As this standardization procedure removes the observed asymmetry inherent to multilevel regression, both models (predicting PA on NA and NA on PA) produce identical results.

In all models, the fixed effects represent the average observed correlation in the sample. Adding participants' grand-mean standardized CES-D scores at the person-level indicates how the relation between PA and NA varies as a function of between person-differences in depressive symptoms.

Supp. Table 1. Multilevel analyses for Study 1 modelling the relation between momentary PA and NA as a function of depressive symptom severity.

$\beta \quad$ SE $\quad t \quad p$

\begin{tabular}{lllll}
\hline Model 1 - predicting standardized NA & & & & \\
Level 1: Standardized PA & -0.503 & 0.021 & -23.858 & $<.001$ \\
Level 2: CES-D & -0.071 & 0.021 & -3.384 & .001 \\
\hline Model 2 - predicting standardized PA & & & & \\
Level 1: Standardized NA & -0.502 & 0.021 & -23.904 & $<.001$ \\
Level 2: CES-D & -0.071 & 0.021 & -3.420 & .001 \\
\hline
\end{tabular}

Supp. Table 2. Multilevel analyses for all three waves in Study 2 modelling the relation between momentary PA and NA as a function of depressive symptom severity.

$\beta \quad$ SE $t \quad p$

Model 1 - predicting standardized NA (Wave 1)
$\begin{array}{lllll} \\ \text { Level 1: Standardized PA } & -0.482 & 0.015 & -32.049 & <.001 \\ \text { Level 2: CES-D } & -0.048 & 0.015 & -3.165 & .002\end{array}$

\section{Model 2 - predicting standardized PA (Wave 1)}

Level 1: Standardized NA

$\begin{array}{llll}-0.482 & 0.015 & -32.053 \quad<.001\end{array}$


Level 2: CES-D

$-0.048$

0.015

$-3.165$

.002

\begin{tabular}{lllll}
\hline Model 3 - predicting standardized NA (Wave 2) & & & & \\
$\begin{array}{l}\text { Level 1: Standardized PA } \\
\text { Level 2: CES-D }\end{array}$ & -0.418 & 0.018 & -23.568 & $<.001$ \\
& -0.033 & 0.018 & -1.846 & .065 \\
\hline Model 4 - predicting standardized PA (Wave 2) & & & & \\
$\quad$ Level 1: Standardized NA & -0.418 & 0.018 & -23.571 & $<.001$ \\
Level 2: CES-D & -0.033 & 0.018 & -1.842 & .065 \\
\hline Model 5 - predicting standardized NA (Wave 3) & & & & \\
$\quad$ Level 1: Standardized PA & -0.442 & 0.017 & -25.552 & $<.001$ \\
$\quad$ Level 2: CES-D & -0.059 & 0.017 & -3.370 & .001 \\
\hline Model 6 - predicting standardized PA (Wave 3) & & & & \\
$\quad$ Level 1: Standardized NA & -0.442 & 0.017 & -25.554 & $<0.001$ \\
\hline Level 2: CES-D & -0.059 & 0.017 & -3.369 & .001 \\
\hline
\end{tabular}

Supp. Table 3. Multilevel analyses for Study 3 modelling the relation between daily sadness and anhedonia as a function of depressive symptom severity.

$\beta \quad$ SE $\quad t \quad p$

\begin{tabular}{lcccc}
\hline Model 1 - predicting standardized sadness & & & & \\
Level 1: Standardized anhedonia & 0.485 & 0.023 & 20.726 & $<.001$ \\
Level 2: CES-D & 0.087 & 0.023 & 3.702 & $<.001$ \\
\hline Model 2 - predicting standardized anhedonia & & & & \\
Level 1: Standardized sadness & 0.485 & 0.023 & 20.719 & $<.001$ \\
Level 2: CES-D & 0.087 & 0.023 & 3.712 & $<.001$ \\
\hline
\end{tabular}


Supp. Table 4. PA-NA correlations that did not relate to participants' CES-D scores.

\begin{tabular}{|c|c|c|c|c|c|}
\hline \multirow[b]{2}{*}{ Positive Affect } & \multirow[b]{2}{*}{ Negative affect } & \multicolumn{2}{|c|}{ Study 1} & \multicolumn{2}{|c|}{ Study 2} \\
\hline & & Correlation & $p$-value & Correlation & $p$-value \\
\hline Happy & Angry & -0.205 & 0.046 & -0.059 & 0.163 \\
\hline Happy & Anxious & -0.081 & 0.437 & -0.059 & 0.164 \\
\hline Happy & Stress & -0.100 & 0.337 & -0.077 & 0.065 \\
\hline Happy & $($ Angry + Stress $)$ & -0.152 & 0.141 & -0.099 & 0.018 \\
\hline Happy & (Angry + Anxious) & -0.219 & $\mathbf{0 . 0 3 3}$ & -0.080 & 0.057 \\
\hline Happy & (Anxious + Stress) & -0.152 & 0.182 & -0.086 & 0.040 \\
\hline Happy & $($ Angry + Anxious + Stress $)$ & -0.177 & 0.086 & -0.142 & 0.001 \\
\hline Relax & Anxious & -0.157 & 0.130 & -0.056 & 0.182 \\
\hline (Happy + Relaxed) & Anxious & -0.129 & 0.214 & -0.067 & 0.111 \\
\hline (Happy + Relaxed) & Stress & -0.172 & 0.094 & -0.130 & 0.002 \\
\hline
\end{tabular}

Note. PA-NA correlations that did correlate in a study are bolded. 
Supp. Table 5. Between-person correlations among all measures across all waves.

\section{Correlations}

\begin{tabular}{llllllllllllllllllllllllll}
\hline 1 & 2 & 3 & 4 & 5 & 6 & 7 & 8 & 9 & 10 & 11 & 12 & 13 & 14 & 15 & 16 & 17 & 18 & 19 & 20 & 21 & 22 & 23 & 24 & 25 & 26
\end{tabular}

\section{CES-D (W1)}

2. DASS-21 depression (W1) .58***

3. DASS-21 anxiety (W1) . $30^{* * * * .47 * * *}$

4. DASS-21 stress (W1) $\quad .51^{* * * * .58^{* * * *} .54^{* * *}}$

5. Mean momentary PA (W1) $\quad-.45^{* * * *-.35^{* * *}-.18^{*}}-. .32^{* * *}$

6. Mean momentary NA (W1) $.42^{* * * *} .45^{* * * *} .38^{* * * *} .45^{* * * *}-.43^{* * *}$

7. SD momentary PA (W1) $\quad .25^{* * *} .27 * * * .14^{*} \quad .32^{* * * *}-.20^{* * *} .28^{* * * *}$

8. SD momentary NA (W1) . $35^{* * * *} .40^{* * * *} .34^{* * * *} .42^{* * * *}-.33^{* * * *} .70^{* * * *} .63^{* * * *}$

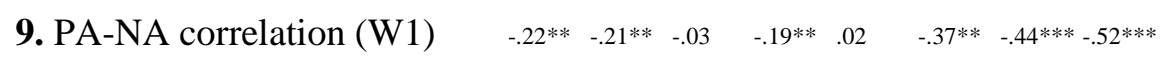

10. CES-D (W2) $\quad .50 * * * .49 * * * .35 * * * .44 * *-.28 * * * .38 * * * .25 * * .33 * * *-.15 *$

11. DASS-21 depression (W2) $.39^{* * * * *} .50^{* * * *} .26^{* * * *} .36^{* * * *}-.22 * * .26^{* * * *} .15^{*} \quad .26^{* * *}-.11 \quad .63^{* * * *}$

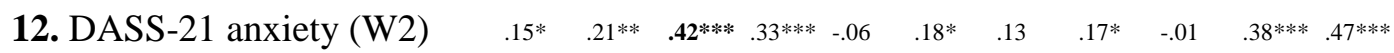

13. DASS-21 stress (W2) $\quad .30^{* * * *} .27^{* * * *} .25^{* * * *} .53^{* * * *}-.15^{*} \quad .34^{* * * *} .16^{*} \quad .28^{* * * *}-.15^{*} \quad .46^{* * * *} .61^{1 * * * *} .60^{* * * *}$

14. Mean momentary PA (W2) $-.32 * * *-.35 * * *-.13 \quad-.31 * * * .67 * * *-31 * * *-.04 \quad-.20^{* * *}-.09 \quad-.36 * * *-36^{* * * *}-.20^{* * *}-.23^{* * *}$

15. Mean momentary NA (W2) $.21^{* *} \quad .27^{* * *} .24^{* *} \quad .40^{* * * *}-.20^{* * *} .73^{* * * *} .17^{*} \quad .44^{* * * *}-.22^{* * *} .42^{* * * *} .31 * * * .23^{* * * *} .43^{* * * *}-.39^{* * * *}$

16. SD momentary PA (W2) $\quad .08 \quad \begin{array}{llllllllllllll} & .10^{* * *} & .07 & .22 * * & -.06 & .08 & .65^{* * *} & .39^{* * * *}-.10 & .18^{*} & .13 & .14^{*} & .16^{*} & -.16^{*} & .19 *\end{array}$

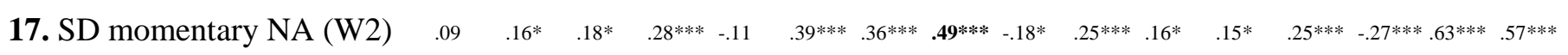

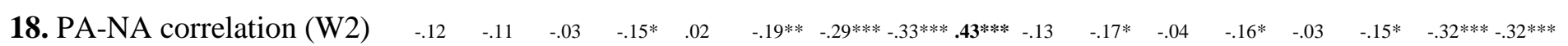

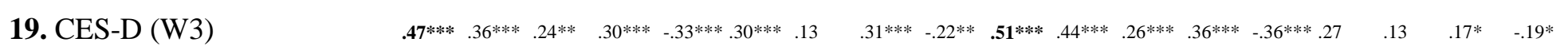

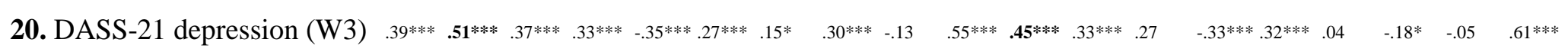

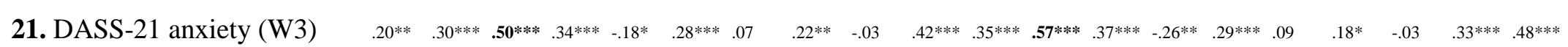

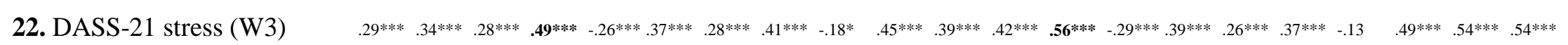

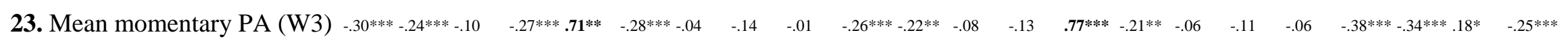

24. Mean momentary NA (W3) $.25^{* * *} .24^{* * * *} .30^{* * * *} .38^{* * *}-.30^{* * * *} .72^{* * * *} .15^{*} \quad .41^{* * * *}-.21^{* * *} .38^{* * *} .24^{* * * *} .20^{* * *} \quad .37^{* * * *}-.30^{* * *} .80^{* * * *} .09 \quad .46^{* * * *}-.04 \quad .35^{* * * *} .42^{* * * *} .30^{* * * *} .42^{* * * *}-.36^{* * * *}$

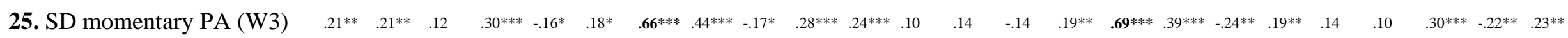

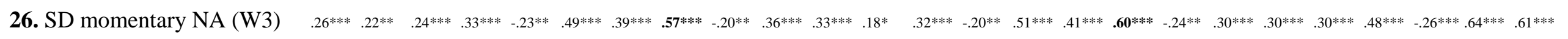

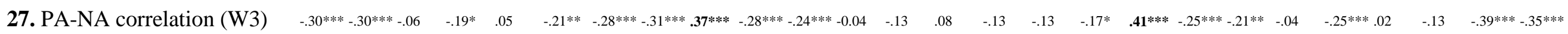
Note. Lagged auto-correlations are bolded. $* p<.050 . * * p<.010 . * * * p<.001$. 
Supp. Table 6. VAR models determining directionality between CES-D scores and PA-NA correlation.

\begin{tabular}{|c|c|c|c|c|}
\hline & $R^{2}(\operatorname{adj})$ & $\beta$ & $t$ & $p$ \\
\hline Model 1 predicting CES-D score at Wave 2 & $.27 * * *$ & & & \\
\hline Intercept & & & 0.53 & .594 \\
\hline CES-D score (Wave 1) & & 0.41 & 5.63 & $<.001$ \\
\hline Mean momentary PA (Wave 1) & & 0.01 & 0.09 & .929 \\
\hline Mean momentary NA (Wave 1) & & 0.22 & 2.85 & .005 \\
\hline PA-NA correlation (Wave 1) & & 0.02 & 0.34 & .733 \\
\hline Model 2 predicting CES-D score at Wave 3 & $.24 * * *$ & & & \\
\hline Intercept & & & 2.28 & .024 \\
\hline CES-D Score (Wave 1) & & 0.37 & 4.88 & $<.001$ \\
\hline Mean momentary PA (Wave 1) & & -0.14 & -1.81 & .072 \\
\hline Mean momentary NA (Wave 1) & & 0.04 & 0.43 & .668 \\
\hline PA-NA correlation (Wave 1) & & -0.12 & -1.67 & .096 \\
\hline Model 3 predicting CES-D score at Wave 3 & $.30 * * *$ & & & \\
\hline Intercept & & & 3.80 & $<.001$ \\
\hline CES-D score (Wave 2) & & 0.42 & 5.89 & $<.001$ \\
\hline Mean momentary PA (Wave 2) & & -0.21 & -2.97 & .003 \\
\hline Mean momentary NA (Wave 2) & & -0.02 & -0.20 & .838 \\
\hline PA-NA correlation (Wave 2) & & -0.15 & -2.28 & .024 \\
\hline Model 4 predicting PA-NA correlation at Wave 2 & $.17 * * *$ & & & \\
\hline Intercept & & & -0.65 & .516 \\
\hline PA-NA correlation (Wave 1) & & 0.41 & 5.64 & $<.001$ \\
\hline Mean momentary PA (Wave 1) & & -0.05 & -0.62 & .537 \\
\hline Mean momentary NA (Wave 1) & & -0.04 & -0.54 & .593 \\
\hline CES-D score (Wave 1) & & -0.03 & -0.40 & .686 \\
\hline
\end{tabular}




\begin{tabular}{lcccc}
\hline Model 5 predicting PA-NA correlation at Wave 3 & $.17 * * *$ & & & \\
Intercept & & -0.16 & .875 \\
PA-NA correlation (Wave 1) & $\mathbf{0 . 3 1}$ & $\mathbf{4 . 1 8}$ & $<.001$ \\
Mean momentary PA (Wave 1) & -0.10 & -1.26 & .210 \\
Mean momentary NA (Wave 1) & -0.02 & -0.28 & .777 \\
CES-D score (Wave 1) & $\mathbf{- 0 . 2 7}$ & $\mathbf{- 3 . 3 4}$ & $<.001$ \\
\hline Model 6 predicting PA-NA correlation at Wave 3 & & & & \\
Intercept & & -1.84 & .067 \\
PA-NA correlation (Wave 2) & & $\mathbf{0 . 3 8}$ & $\mathbf{5 . 6 0}$ & $<.001$ \\
Mean momentary PA (Wave 2) & -0.01 & -0.13 & .900 \\
Mean momentary NA (Wave 2) & 0.03 & 0.42 & .673 \\
CES-D score (Wave 2) & & $\mathbf{- 0 . 2 6}$ & $\mathbf{- 3 . 3 6}$ & $<.001$ \\
\hline
\end{tabular}

$\overline{\text { Note. All } p \text {-values presented are non-corrected for familywise error rate. Effects remaining significant }}$ after a Bonferroni correction are bolded. $* p<.050 . * * p<.010 . * * * p<.001$. 